\title{
Some generalizations of the retract theorem of $T$. Ważewski with applications to ordinary and partial differential equations of the first order
}

\author{
by ANdrzes Pelczar (Kraków)
}

\begin{abstract}
There are given some extensions and gencralizations of the classical retract theorem of $T$. Ważewski, in various forms, for generalized semidynamical systems and certain applications of them in the theory of ordinary and partial differential equations of the first order.

By a generalized semidynamical system we mean a triple $(X, T, \pi)$, where $X$ is a topological space, $T$ is an abelian, ordered semigroup, satisfying some additional conditions, and $\pi: T \times X \rightarrow X$ is a continuous mapping, such that the family $\left\{\pi_{t}\right\}_{t \in T}$ of mappings $\pi_{t}: X{ } x \mapsto \pi(t, x) \in X$ have the semigroup structure with respect to the composition.

The general theory is given in the first part of the paper. Some applications of a local form of the generalized retract theorem in the theory of ordinary equations given as an illustration of the method, and some essentially new results obtained by using the generalized retract theorems, with respect to partial differential equations of the first order, are presented with proofs detaily given, in the second part of the paper.
\end{abstract}

\section{CONTENTS}

Introduction . . . . . . . . . . . . . . . . . . . . . . 16

\section{PART I}

1. Local abelian ordered semigroups . . . . . . . . . . . . . . . 17

2. Generalized semidynamical systems . . . . . . . . . . . . . . . 18

3. Some preliminary lemmas . . . . . . . . . . . . . . . . . . . 20

4. Condition (C) . . . . . . . . . . . . . . . . . . . . . 21

5. Notation . . . . . . . . . . . . . . . . . . . . . . . . 22

6. Invariant sets. Egress and strict egress points . . . . . . . . . . . . . 22

7. A generalization of the Ważewski's retract theorem . . . . . . . . . . 23

8. Continuity of $\sigma$ and some special forms of the retract theorem . . . . . 24

9. Equivalence of Theorems III and $I V^{\prime}$ for compact sets . . . . . . . . . 27 


\section{PA R T II}

1. Investigations of local behavior of solutions of systems of ordinary differential equations . . . . . . . . . . . . . . . . . . . . . . . . . . . . . .

2. Preliminaries necessary for generalized retract theorem for families of surfaces, with applications to partial differential equations. . . . . . . . . 36

3. Applications of general results for families of regular surfaces . . . . . . . 37

4. Applications of the results from Chapter 3 in the theory of partial differential equations of the first order. . . . . . . . . . . . . . . . . . . . .

5. Extensions of the results from Chapter 4 for systems of differential equations

6. Further applications of the main results from the first part in the theory of partial differential equations . . . . . . . . . . . . . . . . . .

\section{INTRODUCTION}

The classical rotract theorem of $T$. Ważewski from [37] and [38] (see also [41], [42]) was extended and generalized by many authors. In particular some general results are given by Albrecht [1], Bielecki and Kluezny [8], Kluczny [14], [15], Fedorov [11], Pelezar [22], [23], [27] Plis [28] (in the paper [28] the concept of quasi-isotopic deformative retract, introduced by $K$. Borsuk in [9] was used). Extensions of the Wazewski's method for contingent equations, and - generally - for multivalued mappings, are due to Bebernes and Schuur [3], [4], Bielecki [7], Pelczar [23]. For references concerning dynamieal systems given by multivalued mappings (in particular by paratingent and contingent equations), we refer to the papers of Bronstein [10] and Roxin [29]. The fundamental applications are given by Wazewski [37], [38] and by the other authors, for instance Kaplan, Lasota and Yorke [13], Fedorov [11], A. Pelczar [24], [25], [26].

There are many papers concerning asymptotic behaviour of solutions of ordinary differential equations of many types, and structures of certain sets generated by solutions having some asymptotic properties. Among them, there are papers using the retract method of T. Wazewski in various version, or some particular notions closely related to this method, for instance the notion of egress points; there are many papers using some other qualitative methods being in close connection with the Ważewski's retract method. We cite only: Kluczny [16], [17], Lojasiewicz [18]. Mikcłajska [19], Szmydt [35], Szarski and Wazewski [34], Tatarkiewicz [36], Olech [20], Onuchic [21], Staikos [31]. The purpose of the present paper is to give a generalization of the classical retract theorem of T. Ważewski, for general semidynamical systems defined by local semigroups of transformations of a topological space into itself. Such generalizations give some possibilities of applications, in particular, in the theory of partial differential equations of the first order. 
The paper has two parts. The first part contains in its 9 sections the general results concerning generalized semidynamical systems. Some of these results were given already (in a little different form) in [22] (see also [23] for additional remarks and a correction to [22]). The second part has 6 chapters; each chapter is divided into sections, some sections are divided into subsections. In the second part we give applications of general results in the theory of differential equations. In the first chapter we give an application for (local) investigation of the behaviour of solutions of ordinary differential equations; there are no essentially new results, but only an example of applications of general results in a local form. Chapters 2-5 contain the results concerning families of regular surfaces, with particular applications in the theory of partial differential equations of the first order. Some of these results are signalized (without details) in [24]. The last Chapter 6 contains some other applications of the main results from the first part, in the investigations of the behaviour of solutions of partial differential equations.

The author is indebted to Professor T. Ważewski for giving helpful ideas while preparing this thesis and many valuable remarks; in particular the author would like to underline that Theorem 8 in Chapter 6 was stated owing to suggestions of Professor T. Ważewski.

The author would like to express his sincere thanks to Professors J. Szarski, A. Plis and D. Bushaw for their valuable advice and remarks.

\section{PART I}

\section{LOCAL ABELIAN ORDERED SEMIGROUPS}

Let $(T, \leqslant)$ be an ordered space (that is: " $\leqslant$ " is a transitive, reflexive and antisymmetric binary relation, and for every $s, t \in T: s \leqslant t$ or $t \leqslant s$ ), having the minimal element 0 .

For $s, t \in T$ (such that $s \leqslant t$ or $s<t$ if it will be necessary for the correctness of definitions of corresponding sets), we put:

$$
\begin{array}{ll}
{[s, t] \stackrel{\text { df }}{=}\{u \in T: s \leqslant u \leqslant t\},} & (s, t) \stackrel{\text { df }}{=}\{u \in T: s<u<t\}, \\
{[s, t] \stackrel{\text { df }}{=}\{u \in T: s<u \leqslant t\},} & {[s, t) \stackrel{\text { df }}{=}\{u \in T: s \leqslant u<t\} .}
\end{array}
$$

By $\langle\cdot, \cdot\rangle$ (or $\langle\cdot, \cdot, \cdot\rangle)$ we denote elements of cartesian products; thus $\langle s, t\rangle$ denotes (for $s, t \in T$ of course) the pair belonging to $T \times T$.

Definition 1 . We say that $(\mathscr{N},+)$ definies in $T$ the local abelian semigroup structure (in this case $(T, \leqslant, \mathscr{N},+$ ) will be said to be a local abelian ordered semigroup, shortly LAO-semigroup) if and only if the following seven conditions hold: 
$\left(\mathrm{C}_{1}\right) \quad \mathscr{N} \subset T \times T$,

$\left(\mathrm{C}_{2}\right) \quad+: \mathscr{N} \geqslant\langle t, s\rangle \mapsto t+s \stackrel{\mathrm{d} t}{=}+(\langle t, s\rangle) \epsilon T$,

$\left(\mathrm{C}_{3}\right)$ for every $t \in T$, there is $s \in T, s>0$, such that $[0, t] \times[0, s] \subset \mathcal{N}$,

$\left(\mathrm{C}_{4}\right) \quad$ if $\langle t, s\rangle \in \mathscr{N}$, then $\langle s, t\rangle \in \mathscr{N}$ and $t+s=s+t$,

$\left(\mathrm{C}_{s}\right)$ if $\langle t, s\rangle,\langle t+s, u\rangle \in \mathscr{N}$, then $\langle t, s+u\rangle,\langle t+u, s\rangle \in \mathcal{N}$ and $(t+s)+$ $+u=t+(s+u)$

$\left(\mathrm{C}_{6}\right)\langle 0, t\rangle \in \mathcal{N}$ and $0+t=t$ for every $t \in T$,

$\left(\mathrm{C}_{7}\right) \quad$ if $\langle t, s\rangle,\langle t, u\rangle \in \mathscr{N}$ and $s \leqslant u$, then $t+s \leqslant t+u$.

Remark 1a. If $\mathscr{N}=T \times T$ fulfils the above conditions $\left(\mathrm{C}_{1}\right)-\left(\mathrm{C}_{7}\right)$, then $(T, \leqslant,+)$ is an ordered abelian semigroup with the neutral element 0 , being also the minimal element. Hence the notion of LAO-semigroup is a generalization of the notion of ordered abelian semigroups. In the other words, $(T, \leqslant, T \times T,+)$ can be identified with an abelian ordered semigroup $(T, \leqslant,+)$.

On the other hand we have the following

Remark $1 \mathrm{~b}$. If $(T, \leqslant,+)$ is an abelian, ordered, dense semigroup without a maximal element, having the neutral element 0 , which is also the minimal element, and condtion (*) (see Section 2, below) is satisfied, and, moreover, $w^{0}>0$ is a fixed element of $T$, then putting $T^{0}$ $=\left[0, w^{0}\right)$ and $\mathscr{N}^{0}=\left\{\langle s, t\rangle: s, t, s+t \epsilon T^{0}\right\}$ we obtain a LAO-semigroup $\left(T^{0}, \leqslant^{0}, \mathscr{N}^{0},+^{0}\right)$, where $\leqslant^{0}$ and $+^{0}$ are the natural restrictions of $\leqslant$ and + to $T^{0}$ and $T^{0} \times T^{0}$ respectively.

An important example of such a situation is the case: $T=R_{*}$ $=[0, \infty)$ with $T^{0}=\left[0, w^{0}\right)$, where $w^{0}$ is a positive real number, and + and $\leqslant$ are the usual addition and ordering relation in real number space.

\section{GENER ALIZED SEMIDYNAMICAL SYSTEMS}

Let $X$ be a topological space and let $(T, \leqslant, \mathscr{N},+)$ be a LAO-semigroup (with some $\mathscr{N} \subset T \times T$ ) having the neutral element 0 , being also the minimal element of $T$, without a maximal element, such that for every $S \subset T, S \neq \emptyset$, inf $S$ exists. We assume that $(T, \leqslant)$ is dense: for every $s, t \in T, s<t$, there exists a $u \in T$ such that $s<u<t$. We will consider $T$ also as a topological space (but not necessarily as a topological semigroup!) with the natural topology given by the ordering relation; this means that the basis of neighbourhoods for any $t^{0} \epsilon T, t^{0}>0$ is the family $\left\{(s, t): s<t^{0}<t\right\}$ and the basis of neighbourhoods of 0 , is the family $\{[0, t): t>0\}$. We will assume the following condition:

(*) $\quad(s, t \in T, s<t) \Rightarrow$ (there exists a $\lambda \in T, \lambda>0$, such that $s+\lambda \leqslant t)$. 
Remark 2 . The density of $(T, \leqslant)$ and condition (*) imply immediately that:

(**) $\quad(s, t \in T, s<t) \Rightarrow($ there exists a $\lambda \in T, \lambda>0$, such that $s+\lambda<t)$.

Conversely, condition $(* *)$ implies trivially condition $(*)$.

ASSUMP'IION. Throughout the whole first part of the present paper, $X$ and $(T, \leqslant, \mathscr{N},+)$ will be as above; we shall consider them as fixed in the sequel.

Definition 2. Suppose that $T^{0}=T$ or $T^{0}=\left[0, v^{0}\right)$, where $w^{0} \epsilon T, w^{0}$ $>0$, and that $T \times T \subset \mathcal{N}$ in the first case (and observe that obviously $\{0\} \times\left[0, w^{0}\right) \subset \mathscr{N}$ in the second case).

A mapping $\pi: T^{0} \times X \rightarrow X$ is said to be of the class $\mathscr{G}\left(T^{0}\right)$ (or shortly: $\left.\pi \in \mathscr{G}\left(T^{0}\right)\right)$ if and only if

$\left(G_{1}\right) \pi$ is continuous as a mapping from the topological space $T^{0} \times X$ provided with the natural topology of the cartesian product given in the usual way by the topology on $T^{0}$ (induced by the topology on $T$ ) and $X$, into the topological space $X$;

$\left(\mathrm{G}_{2}\right)$ the family $\left\{\pi_{t}: t \in T^{0}\right\}$, where for every $t \in T^{0}$

$$
\pi_{t}: X \ni x \mapsto \pi_{t}(x) \stackrel{\text { df }}{=} \pi(t, x) \epsilon X
$$

fulfils the following (local) semigroup conditions:

$$
\begin{gathered}
\pi_{s} \circ \pi_{t}=\pi_{t} \circ \pi_{s}=\pi_{t+s} \quad \text { for every } s, t \epsilon T^{0},\langle s, t\rangle \epsilon \mathscr{N}, \\
\pi_{0}=i d_{X}\left(\Leftrightarrow \pi_{0}(x)=x \text { for every } x \epsilon X\right) .
\end{gathered}
$$

If - in some particular questions - the set $T^{0}$ will be fixed, then $\mathscr{G}\left(T^{0}\right)$ will be denoted shortly by $\mathscr{G}$.

Definition 3. For every $\pi \epsilon \mathscr{G}\left(T^{0}\right)$, we will call the triple $\left(X, T^{0}, \pi\right)$ a generalized semidynamical system (GSD-system).

Remark 3. Perhaps a natural name for triples $\left(X, T^{0}, \pi\right)$ with $\pi$ belonging to $\mathscr{G}$, would be local semidynamical systems. But Bhatia and Hajek [5] have already used the name "local semidynanical systems" for some other systems, and therefore to avoid confussion, we will use here a terminology slightly different from that used in [5]. Note, that GSD-systems considered here are special cases of local semidynamical systems from [5] if $T=R_{*}$.

Remark 4. In the present paper we will prove general theorems concerning GSD-systems for arbitrary $T$ (under assumptions introduced above, of course). However, applications given in the second part of the paper concern only the case, namely $T=R_{*}=[0, \infty)$. Hence, from the 
practical point of view, one can think always about $T$ being the real halfline. An example of $T$ being not the positive real half-line, and an application of the general results presented here for such strange $T$, is given in $[21]$.

\section{SOME PRELIMINARY LEMMAS}

Lemua 1. If $T^{0}=\left[0, w^{0}\right)$ or $T^{0}=T$ and the mapping $\lambda: T^{0} \rightarrow X$ is continuous, $M \subset X, t, t^{\prime} \in T^{0}, t<t^{\prime}, \lambda(t) \in M, \lambda\left(t^{\prime}\right) \&$, then putting

$$
S=\left\{s \in\left[t, t^{\prime}\right]: \lambda(s) \notin M\right\} \quad \text { and } \quad s^{0}=\inf S,
$$

we have

$$
\lambda\left(s^{0}\right) \in \partial M \quad(=\text { the boundary of } M) .
$$

Proof. Let $U \subset X$ be a neighbourhood of $\lambda\left(s^{0}\right) \epsilon X$. Since $\lambda$ is continuous, there is a neighbourhood $Q \subset T^{0}$ of $s^{0}\left(Q=(u, v)\right.$ with some $u, v \epsilon T^{0}$, $u<s^{0}<v$ if $s^{0}>0$ and $Q=[0, v)$ with some $v>0$, if $s^{0}=0$ ), such that $\lambda\left(Q \cap\left[t, t^{\prime}\right]\right) \subset U$. Since $s^{0}=\inf S$, we have $S \cap Q \cap\left[t, t^{\prime}\right] \neq \emptyset$ and then $U \cap(X \backslash M) \neq \emptyset$. On the other hand $Q \cap\left[t, t^{\prime}\right] \cap\left(T^{0} \backslash S\right) \neq \emptyset$ (if not, there will exist an element $s \in S$ such that $s<s^{0}$ ), and then we have: $U \cap M \neq \emptyset$. The neighbourhood $U$ of $\lambda\left(s^{0}\right)$ was arbitrarily fixed. Then, we have proved, that for every neighbourhood $U$ of $\lambda\left(s^{0}\right)$ we have: $U \cap J I$ $\neq \emptyset$ and $U \cap(X \backslash M) \neq \emptyset$. This means that $\lambda\left(s^{0}\right) \epsilon \bar{M} \cap(\overline{X \backslash M})$ and then $\lambda\left(s^{0}\right) \epsilon \partial \mu$.

LeMma 2. For every $t_{1}, t_{2} \in T, t_{1} \leqslant t_{2}$, the interval $\left[t_{1}, t_{2}\right]$ is compact.

Proof. We will repeat here the classical reasoning showing that a closed and bounded interval in $R=(-\infty,+\infty)$ is compact. Let $\left[t_{1}, t_{2}\right] \subset T$ be fixed and let $\mathscr{H}$ be a family of open sets covering $\left[t_{1}, t_{2}\right]$, that is such that $\left[t_{1}, t_{2}\right] \subset \bigcup\{H \in \mathscr{H}\}$. Suppose that there are no finite subfamilies of $\mathscr{H}$ covering $\left[t_{1}, t_{2}\right]$. Thus, the set $P=\left\{t \in T: t_{1} \leqslant t \leqslant t_{2}\right.$, $\left[t_{1}, t\right]$ is not contained in the union of any finite subfamily of $\left.\mathscr{H}\right\}$ is nonempty. Put $s=\inf P$. Let $H^{0}$ be such that $s \in H^{0} \epsilon \mathscr{H}$. Since $H^{0}$ is open, there exist $s^{\prime}, s^{\prime \prime} \in T$, such that $s^{\prime}<s<s^{\prime \prime}$ and $\left(s^{\prime}, s^{\prime \prime}\right) \subset H^{0}$. Consider $\left[t_{1}, s\right]$. Because of the definition of $s=\inf P$, for every $s \in T$ such that $s \in\left[t_{1}, s\right)$ there exists a finite subfamily of $\mathscr{H}$ of open sets, say $H^{1}, \ldots, H^{\prime \prime}$, such that $\left[t_{1}, \hat{s}\right] \subset \bigcup\left\{H^{i}: i=1, \ldots, p\right\}$. Hence, for $\hat{s} \in\left[t_{1}, s\right) \cap\left(s^{\prime} s^{\prime \prime}\right)$, we have:

$$
\left[t_{1}, s\right] \subset\left[t_{1}, \hat{s}\right] \cup\left(s^{\prime}, s^{\prime \prime}\right) \subset \bigcup\left\{H^{i}: i=0,1, \ldots, p\right\} .
$$

Thus, we have proved, that $\left[t_{1}, s\right]$ can be covered by a finite subfamily of $\mathscr{H}$. This, in virtue of the assumption that the set $P$ is non-empty, yields us the inequality $s<t_{2}$. Let us consider again the set $B^{0} \epsilon \mathscr{H}$, such that $s \in H^{0}$. There are $s^{\prime}, s^{\prime \prime} \in T$, such that $s \in\left(s^{\prime}, s^{\prime \prime}\right),\left(s^{\prime}, s^{\prime \prime}\right) \subset H^{0}$ and $s^{\prime \prime}<t_{2}$. As previously, take $\hat{s} \epsilon\left(s^{\prime}, s^{\prime \prime}\right) \cap\left[t_{1}, s\right)$. Take also a point $\check{s} \epsilon\left(s, s^{\prime \prime}\right)$. 
We have

$$
\left[t_{1}, \check{s}\right] \subset\left[t_{1}, \hat{s}\right] \cup\left(s^{\prime}, s^{\prime \prime}\right) \subset \bigcup\left\{H^{i}: i=0,1, \ldots, p\right\}
$$

which is impossible in virtue of the definition of $s=\inf P$, because $\check{s}>\boldsymbol{s}$.

CoRollary. Every closed interval $[s, t] \subset T^{0}$ is compact in $T^{0}$, with respect to the topology induced in $T^{0}$ by the topology of $T$.

\section{CONDITION (C)}

Definition 4. Let $M$ be an open subset of $X, M \neq \emptyset$, let $w^{0} \epsilon T$, $w^{0}>0$ be fixed, and let $\pi$ be a mapping from $\left[0, w^{0}\right) \times X$ into $X$ (resp. from $T \times X$ into $X$ ). We say that $M$ fulfils condition $C\left(w^{0} ; \pi\right)$ (resp. $C(\pi)$ ) if and only if, for every $t, t^{\prime} \in\left[0, w^{0}\right)$ (resp. $t, t^{\prime} \in T$ ) such that $t<t^{\prime}$ and every $x \in M$ such that $\pi(s, x) \epsilon M$ for $0 \leqslant s \leqslant t^{\prime}$, there exists a neighbourhood $W$ of the point $x$ such that

$$
y \in W \Rightarrow \pi(s, y) \in M \quad \text { for } 0 \leqslant s \leqslant t .
$$

We say that $\pi$ fulfils cordition $\left(C\left(w^{0}\right)\right)$ (resp. $(C)$ ) if and only if condition $C\left(w^{0} ; \pi\right)$ (resp. $C(\pi)$ ) is satisfied for each open and non-empty subset $\boldsymbol{I}$ of $\boldsymbol{X}$, that is for every $\left\langle x, t, t^{\prime}, M\right\rangle \epsilon X \times \boldsymbol{T} \times \boldsymbol{T} \times \mathcal{O}(X)$, where $\mathcal{O}(X)$ denotes the fanily of all open and non-empty subsets of $X$, such that $0 \leqslant t<t^{\prime}<w^{0}$ (resp. $0 \leqslant t<t^{\prime}$ ) and $\pi(s, x) \in M$ for $0 \leqslant s<t^{\prime}$, there exists a neighbour hood $W$ of $x$ such that implication (4) holds true.

Since $M$ is open, $W$ can be required to be a subset of $M$.

Remark 5. In the classical cases of dynamical systems given by systems of differential equations, the usual conditions of regularity assumed there imply conditions (C). In the general theory of generalized dynamical systems, one assumes very often some stronger conditions; for instance, Hale and Infante assume in [12] uniform continuity of $\pi$ in bounded sets. TVe shall prove below a lemma concerning condition (C) in the case of continuous $\pi$; more precisely, we shall prove that (C) is satisfied for $\pi$ belonging to $\mathscr{G}$.

LEMna 3. For every $\pi \in \mathscr{G}=\mathscr{G}\left(T^{0}\right)$, where $T^{0}=\left[0, w^{0}\right)$ or $T^{0}=T$ $\left(w^{0}>0\right)$, condition $\left(C\left(w^{0}\right)\right)$ or $(C)-$ respectively - is satisfied.

Proof. Let $M \subset X$ be open and non-empty. Let $t, t^{\prime} \in T^{0}$ and $x \in M$ be such that $t<t^{\prime}$ and $\pi_{s}(x) \in M$ for $s \in\left[0, t^{\prime}\right)$. Consider the interval $[0, t]$. For every $8 \in[0, t]$ (and for the fixed point $x$ ), there exist neighbourhoods $S_{s, x}=\left(t_{s}^{1}, t_{s}^{2}\right)$ of $s\left(\right.$ or $S_{s, x}=\left[0, t_{s}^{2}\right)$, if $\left.s=0\right)$ and $V_{s, x}$ of the point $x$, such that:

$$
\left(u \in S_{s, x}, y \in V_{s, x}\right) \Rightarrow \pi_{u}(y) \in M .
$$

This is obvious in virtue of the continuity of $\pi$. 
Of course, the family of open sets $\left\{S_{s, x}\right\}_{s \varepsilon[0, t]}$ covers the interval $[0, t]$. Since $[0, t]$ is compact (see Lemma 2), we can choose a finite subfamily $\left\{S_{s_{i}, x}\right\}_{i=1, \ldots, p}$ convering the interval $[0, t]$. Put $V=\bigcap\left\{V_{s_{i}, x}\right.$ : $i=1, \ldots, p\}$. We have the following sequence of implications:

$$
\begin{aligned}
\langle u, y\rangle \in[0, t] \times V & \Rightarrow\langle u, y\rangle \in \bigcup\left\{S_{s_{i}, x}: i=1, \ldots, p\right\} \times V \\
& \Rightarrow\langle u, y\rangle \in S_{s_{j}, x} \times V \text { for some } j \epsilon\{1, \ldots, p\} \\
& \Rightarrow\langle u, y\rangle \epsilon S_{s_{j}, x} \times V_{s_{j}, x} \text { for some } j \epsilon\{1, \ldots, p\} \\
& \Rightarrow \pi_{u}(y) \in M .
\end{aligned}
$$

Thus, if $y \in V$, then $\pi_{u}(y) \in M$ for $u \in[0, t]$.

CoRollaRy. For every open set $M \neq \emptyset$, and every $\pi \in \mathscr{G}\left(T^{0}\right)$, the condition $C\left(w^{0} ; \pi\right)$ is satisfied.

\section{NOTATION}

Let $\left(X, T^{0}, \pi\right)$ be a GSD-system (with $T^{0}=\left[0, w^{0}\right)$ ). Let $\pi \epsilon \mathscr{G}=\mathscr{G}\left(T^{0}\right)$ and let $y \in X$.

We put

$$
\begin{aligned}
\pi(y) & =\left\{\pi_{t}(y): t \in T^{0}\right\} \\
\pi^{-1}(y) & =\{z \in X: y \in \pi(z)\} .
\end{aligned}
$$

If $z \in \pi^{-1}(y)$, then we define:

$$
\begin{gathered}
t(z, y)=\inf \left\{t \in T^{0}: \pi_{t}(z)=y\right\}, \\
(z, y)=\left\{\pi_{t}(z): 0<t<t(z, y)\right\}, \\
{[z, y)=(z, y) \cup\{z\} \quad\left(=\left\{\pi_{t}(z): 0 \leqslant t<t(z, y)\right\}\right) .}
\end{gathered}
$$

The same notation will be used in the case of GSD-systems $(x, T, \pi)$, where $T$ is a semigroup.

\section{INVARIANT SETS. EgRESS AND STRICT EgRESS POINTS}

Let $\left(X, T^{0}, \pi\right)$ be a GSD-system and let $N \subset X$ be non-empty.

Definition 5. We say that $N$ is invariant (with respect to $\pi$ ) if and only if $\pi(y) \subset N$ for every $y \in N$.

Definition 6. Let $M \subset X$ be open and non-empty. We say that $y \epsilon \partial M$ is an egress point if and only if there exists $z \epsilon \pi^{-1}(y) \cap M$, such that $[z, y) \subset M$. The point $y \in \partial M$ is said to be a strict egress point if and only if $y$ is an egress point, and for every $\lambda \in T^{0}, \lambda>0$, there is $\eta \in T^{0}$, $\eta<\lambda$, such that $\left(y, \pi_{\eta}(y)\right) \neq \emptyset$ and $\bar{M} \cap\left(y, \pi_{\eta}(y)\right)=\emptyset$. 
By $M_{e}$ and $M_{s e}$ we will denote the sets of all egress and all strict egress points respectively.

Remark 6. The definition of a strict egress point can be given equivalently in the following, slightly modified form: $y \epsilon \partial M$ is a strict egress point if and only $y \in M_{e}$ and there is an $\eta \in T^{0}, \eta>0$, such that $\left(y, \pi_{\lambda}(y)\right)$ $\neq \emptyset$ for $0<\lambda \leqslant \eta$ and $\bar{M} \cap\left(y, \pi_{\eta}(y)\right)=\emptyset$.

Remark 7. Suppose that $M \subset X$ is open and non-empty. Then the following three conditions (a), (b), (c) are equivalent: (a) $y \in \boldsymbol{M}_{e}$, (b) for every open and invariant set $N \subset X$ such that $M \subset N, y \in \partial M \cap N$ and there is an $z \in \pi^{-1}(y) \cap M$ for which $[z, y) \subset M$, (c) there exists an open and invariant set $N \subset X$, containing $M$, such that $y \in \partial M \cap N$ and there is an $z \in \pi^{-1}(y) \cap M$, for which $[z, y) \subset M$.

In book [6] the definition of egress points is given in a form similar to (c). Our first definition is an extension of the original definition of Ważewski [33], [34], by using the notation similar to that of [6].

Remark 8. Let $M \subset N \subset X$ be open, $N$ be invariant, $M \neq \emptyset$ and let $\pi \epsilon \mathscr{G}=\mathscr{G}\left(T^{0}\right)$ (in particular, $N$ can be equal to $X$ ). If $\partial M \cap N \cap M_{e}=\emptyset$, then $M$ is invariant. Indeed, if $M$ is not invariant, then there is $y \in M$, such that $\pi(y) \notin M$. Hence, there is $t \epsilon T^{0}$, such that $\pi_{i}(y)$ does not belong to $M$. Put $\sigma=\inf \left\{t \epsilon T^{0}: \pi_{i}(y) \& M\right\}$. We have $\pi_{\sigma}(y) \epsilon \partial M$ (see Lemma 1). Since $N$ is invariant and $y \in N$, we have $\pi_{\sigma}(y) \in N$. Putting $w=\pi_{\sigma}(y)$, we have $\sigma=t(y, w)$. Hence $[y, w) \subset M$. Thus $w \in M_{e}$. Then, we have proved, that there is $\mathbb{w} \epsilon M_{e} \cap N \cap \partial M$; this is impossible because of the assumption.

The above remark generalizes a lemma from [6] (p. 96) and gives some information about of properties of $M_{e}$.

\section{A GENERALIZATION OF THE WAŹEWSKIS RETRACT THEOREM}

Proposition 1. If $M \subset X$ is open and non-empty, $S \subset M \cup \partial M$ is non-empty, $\pi \in \mathscr{G}\left(T^{0}\right)$ and

$$
S^{\prime}=\{y \in S: \pi(y) \notin M\}
$$

is non-empty, then for every $y \in S^{\prime}$

$$
\sigma(y) \stackrel{\text { df }}{=} \inf \left\{t \in T^{0}: \pi_{t}(y) \in \partial M\right\}
$$

is a well defined element of $T^{0}$ (see Lemma 1). Then we can define the following mapping

$$
S^{\prime} \rightarrow y \mapsto \sigma(y) \in T^{0} .
$$

Moreover, if $y \in S^{\prime} \cap M$, then $\sigma(y)=\inf \left\{t \in T^{0}: \pi_{i}(y) \in M_{e}\right\}$ and $\pi_{\sigma(\nu)}(y) \in M_{e}$. If $y \in S^{\prime} \cap \partial M$, then $\sigma(y)=0$. 
Theonem I. Let $\left(X, T^{0}, \pi\right)$ be a GSD-system, let $M \subset X$ be open and non-empty, $B$ be a subset of $\partial M$ (the case $B=\emptyset$ is not excluded). Suppose that $S \subset M \cup M_{e} \cup B$ is non-empty, such that there is a retraction $\left(M_{e} \cup(B \cap S)\right)$ $\rightarrow S \cap\left(M_{e} \cup B\right)$, but there are no retractions $S \rightarrow S \cap\left(M_{e} \cup B\right)$. Suppose, moreover, that if $S^{\prime}$ defined by (10) is non-empty, then the mapping $f: S^{\prime}{ }_{3} x$ $\mapsto \pi_{\sigma(x)}(x) \in M \Gamma_{e} \cup B$, where $\sigma(x)$ is given by (11), is continuous.

Cnder the above assumptions, there is a $y \in S \cap M$, such that $\pi(y) \subset M$.

Proof. Suppose that the set $\{y \in S: \pi(y) \subset M\}$ is enpty. Then $S=S^{\prime}$, and $f$ is defined and continuous in $S$. The mapping $h=g \circ f$, where $g: M_{e} \cup(B \cap S) \rightarrow S \cap\left(M_{e} \cup B\right)$ is a retraction, is evidently a retraction $S \rightarrow S \cap\left(M_{e} \cup B\right)$. This, however, contradicts the assumptions.

The above theorem is a generalization of the retract theorem of T. Wazewski in the version of [38]. A generalization of another form of the retract theorem (cf. [37]) will be given below.

A special case of Theorem $I$, is the following

Theorem Ia. Let $\left(X, T^{0}, \pi\right)$ be a GSD-system, $M \subset X$ be open, $M \neq \emptyset$, $a$ set $S \subset M \cup \boldsymbol{I}_{e}$ be non-empty and such that there is a retraction $M_{e} \rightarrow$ $S \cap M_{e}$, but there are no retractions $S \rightarrow S \cap M_{e}$. Suppose that if $S^{\prime}$ defined by (10) is non-empty, then the mapping $f: S^{\prime}{ }_{3} x \mapsto \pi_{\sigma(x)}(x) \in M_{e}$ is continuous. Then there exists a $y \in S \cap M$, such that $\pi(y) \subset M$.

This form of the retract theorem is presented in [20] as Theorem $\mathbf{A}^{\prime \prime}$.

\section{CONTINUITY OF $\sigma$ AND SOME SPECIAL FORM OF THE RETRACT THEOREM}

It is easy to see that a fundamental role in Theorem $I$ is played by the continuity of $\sigma$. Indeed, the mapping $f$ introduced in the assumptions of Theorem $I$ is equal to $\pi \circ(\sigma$, identity $)$ and then it is continuous if $\sigma$ is continuous. Thus, it is very important for applications to give some conditions sufficient for $\sigma$ to be continuous.

Let us put, for non-empty open $M \subset X$ :

$$
\begin{gathered}
M^{*} \stackrel{d f}{=}\left\{y \in \partial M: \text { for every } \lambda \in T^{0}, \lambda>0, \text { there exists } \eta \in T^{0}, \eta<\lambda,\right. \\
\text { such that } \left.\left(y, \pi_{\eta}(y)\right) \neq \emptyset \text { and } \bar{M} \cap\left(y, \pi_{\eta}(y)\right)=\emptyset\right\} .
\end{gathered}
$$

Remark 9. For a set $N \subset X$ define $N$-start points as follows: $x \in N$ is said to be an $N$-start point if and only if there are no $y \in N \backslash\{x\}$ such that $x \in \pi(y)$ (in the other words: $\pi^{-1}(x) \cap N=\{x\}$ and if $\pi(t, y)=x$, then $t=0$ and $y=x$ or $y \& N)$. This definition is an extension of the definition of start points given by Bajaj [2]. Using this notation, and denoting by $N^{s}$ the set of all $N$-start points, we can state the following obvious relations:

$$
(\bar{M})^{s} \cap M_{e}=\emptyset, \quad M_{s e} \cup(\bar{M})^{s} \subset M^{*} \cup\{x \in \bar{M}: \pi(x)=\{x\}\}, \quad(\bar{M})^{s} \subset(\partial M)^{s} .
$$


In order to underline certain particular properties of some sets, we will use in the sequel the set

$$
M^{\prime} \stackrel{\text { df }}{=}(\bar{M})^{s} \cap M^{*} \quad\left(=\left\{y \in M^{*}: \pi^{-1}(y) \cap \bar{M}=\{y\}\right\}\right) .
$$

Theorem II. Let $\left(X, T^{0}, \pi\right)$ be a GSD-system, $M \subset X$ be open and non-empty such that $M_{c}=M_{s e}$. Suppose that $S \subset M \cup M^{*}$ is non-empty and such that for every $y \in S, \pi(y) \notin M$. Then the mapping $S \ni y \mapsto \sigma(y) \epsilon T^{0}$, with $\sigma$ given by (11), is continuous.

Remark 10. Since $M_{e}=M_{s e}$, we have $M_{e} \cup M^{*}=M_{s e} \cup M^{*}$ $=M^{*} \supset M^{\prime} \cup M_{s e}$ (see Remark 9). An important (for applications) case is $M^{*}=\left[(\bar{M})^{s} \cap M^{*}\right] \cup\left[M_{s e} \cap M^{*}\right]$, which gives $M^{*} \subset(\bar{M})^{s} \cup M_{s e}$. In this case $M^{\prime} \cup M_{s e}=M^{*}$ and then Theorem II can be equivalently stated with $M^{*}$ replaced by $M_{e} \cup M^{\prime}$, or by $M_{e} \cup M^{*}$, or by $M_{s e} \cup M^{\prime}$, or finally by $M_{s e} \cup I^{*}$.

Proof of Theorem II. Let $y \in S$. The have $\pi(y) \notin M$ and then there is a $t \in T^{0}$, such that $\pi_{i}(y) \in \partial M$ (see Lemma 1 in the case $y \in M \cap S$; in the case $y \in M^{*} \cap S$ the existence of such $t$ is trivial, since $\pi_{0}(y)$ $\left.=y \in M^{*} \cap S \subset \partial M\right)$. Then there exists a $t \in T^{0}$ such that $\pi_{t}(y) \in M_{e} \cup M^{*}$ $=\boldsymbol{M}_{s e} \cup \boldsymbol{M}^{*}=M^{*}$; if, moreover, $y \in S \backslash M^{*}$, then $\pi_{t}(y) \epsilon M_{e}=M_{s e}$. We have (seo Proposition 1):

$$
\begin{aligned}
\sigma(y) & =\inf \left\{t \in T^{0} ; \pi_{t}(y) \leftrightarrow M\right\}=\inf \left\{t \in T^{0}: \pi_{t}(y) \epsilon \partial M\right\} \\
& =\inf \left\{t \in T^{0}: \pi_{t}(y) \epsilon M_{e}\right\}=t\left(y, \pi_{\sigma(y)}(y)\right) \quad \text { for every } y \in S \cap M,
\end{aligned}
$$

and $\sigma(y)=0$ for $y \in S \cap M^{*} \subset \partial M$.

Let $y$ be a point of $S \backslash M^{*}=S \cap M$. Consider $\sigma(y)$ and an arbitrarily fixed neighbourhood $\left(t_{1}, t_{2}\right) \subset T^{0}$ of $\sigma(y)$. Of course, $0 \leqslant t_{1}<\sigma(y)<t_{2}$. Since $\pi_{\sigma(y)}(y) \in M_{e}=M_{s e}$ and $\sigma(y)=t\left(y, \pi_{\sigma(y)}(y)\right)$, we have $\left[\pi_{t_{1}}(y)\right.$, $\left.\pi_{\sigma(y)}(y)\right) \subset M$ and

$$
\left(\pi_{\sigma(y)}(y), \pi_{s}(y)\right) \neq \varnothing \quad \text { and } \quad\left(\pi_{\sigma(y)}(y), \pi_{s}(y)\right) \cap \bar{M}=\emptyset
$$

for some $s \in T^{0}$, such that $\sigma(y)<s<t_{2}$. Indeed, we can choose $t^{\prime} \epsilon T^{0}$, $t^{\prime}>0$, such that $\sigma(y)+t^{\prime}<t_{2}$ (see condition (*) from the second section). On the other hand, putting $z=x_{\sigma(y)}(y)$, we obtain: $z \in M_{s e}$, and then we can find for the $t^{\prime}$ chosen previously an element $s^{\prime} \in T^{0}$ such that $0<s^{\prime}$ $<t^{\prime},\left\{z, \pi_{s^{\prime}}(z)\right) \neq \emptyset$ and $\left\{z, \pi_{s^{\prime}}(z)\right) \cap \bar{M}=\emptyset$. We have obviously $\pi_{s^{\prime}}(z)$ $=\pi_{s^{\prime}}\left(\pi_{\sigma(y)}(y)\right)=\pi_{\sigma(y)+s^{\prime}}(y)$, and then putting $s=\sigma(y)+s^{\prime}$, we obtain the element $s$ having the required properties (15).

Furthermore, we can require the condition $\left(\pi_{\sigma(y)}(y), \pi_{s}(y)\right] \cap \bar{M}=\emptyset$, a little stronger than the second condition of (15) (if $\pi_{s}(y)$ belongs to $\bar{M}$, then we can replace $s$ by some $\tilde{s} \epsilon(\sigma(y), s)$, for which $\left(\pi_{\sigma(y)}(y), \pi_{\tilde{s}}(y)\right] \cap \bar{M}$ $=\emptyset$ ). 
Since $\pi_{t}(y) \epsilon M$ for $0 \leqslant t<\sigma(y)$, there is a neighbourhood $W$ of the point $y$ such that $W \subset M$ and $\pi_{t}(w) \in M$ for $0 \leqslant t<t_{1}$ and $w \in W$. This follows directly from the condition $C(\pi)$, which is satisfied (see Lemma 3) because $\pi \epsilon \mathscr{G}$.

Moreover, $\pi_{s}(y) \epsilon X \backslash \bar{M}$, and then there is a neighbourhood $V(\subset M)$ of $y$, such that $\pi_{s}(x) \epsilon X \backslash \bar{M}$ for $x \in V$. Then, for any point $x \in W \cap V \cap S$, we have: $\pi_{t}(x) \in M$ for $t \in\left[0, t_{1}\right)$ and $\pi_{s}(x) \notin \bar{M}$.

Hence (see Lemma 1) for $x \in W \cap V \cap S$ we have: $t_{1}<\sigma(x) \leqslant s \leqslant t_{2}$. Because of the relation: $\pi_{s}(x) \notin \bar{M}$, the equality $\sigma(x)=s$ is impossible for $x \in W \cap V \cap S$. Thus, for such $x$, we have $t_{1}<\sigma(x)<t_{2}$. Then, for every neighbourhood $Q$ of $\sigma(y)$, there is a neighbourhood $U(=W \cap V \cap S)$ of $y \in S$, such that

$$
x \in U \Rightarrow \sigma(x) \in Q
$$

This means that $\sigma$ is continuous in the set $S \backslash M^{*}$.

Let now $y \in S \cap M^{*} \subset \partial M$. Then $\sigma(y)=0$ and $\pi_{\sigma(y)}(y)=\pi_{0}(y)=y$. Consider a neighbourhood $\left[0, t_{2}\right) \subset T^{0}$ of $\sigma(y)$. Since $y \epsilon M^{*}$, there is a $s \in T^{0}$ such that $0<s \leqslant t_{2},\left(y, \pi_{s}(y)\right) \neq \varnothing$ and $\left(y, \pi_{s}(y)\right) \cap \bar{M}=\varnothing$ (formally, from the definition of $M^{*}$, we have only $\left(y, \pi_{s}(y)\right] \cap \bar{M}=\varnothing$, but as in the preceding case, we can replace $s$ by some $s^{*} \epsilon(0, s)$ for which the required stronger condition holds true). Hence, in particular, $\pi_{s}(y) \epsilon X \backslash \bar{M}$. From the continuity of $\pi$, it follows that there is a neighbourhood $V$ of $y$ such that $\pi_{a}(x) \in \bar{X} \backslash \bar{M}$ for $x \in V \cap S \subset M \cup M^{*}$. Consider a point $x \in V \cap S$. If $x \in M^{*}$, then $\sigma(x)=0$ and $\sigma(x)$ belongs to the interval $\left[0, t_{2}\right)$ trivially. If $x \notin M^{*}$, then $x \in M$ and then $\sigma(x) \epsilon(0, s) \subset\left[0, t_{2}\right)$. Then, for every neighbourhood $Q$ of $\sigma(y)=0$, there is a neighbourhood $U(=V \cap S)$ of $y$, such that $x \in U \Rightarrow \sigma(x) \in Q$. Thus, $\sigma$ is continuous in $S \cap M^{*}$. Since $S=\left(S \cap M^{*}\right) \cup\left(S \backslash M^{*}\right)$, we have proved the continuity of $\sigma$ in the whole set $\mathbb{S}$.

From Theorems I and II we obtain immediately the following

THeorerr III. Suppose that $\left(X, T^{0}, \pi\right)$ is a GSD-system, $M \subset X$ is non-empty and open, $M_{e}=M_{s e}$. Suppose that $S \subset M \cup \dot{M}^{*}\left(=M \cup M_{e} \cup M^{*}\right)$ is non-empty and such that $S \cap M_{e}\left(=S \cap M_{e} \cap M^{*}\right)$ is a retract of $M_{e} \cup\left(M^{*} \cap S\right)$, but there are no retractions $S \rightarrow S \cap M^{*}\left(=S \cap\left(M_{e} \cup M^{*}\right)\right)$.

Then there exists a $y \in S \cap M$ such that $\pi(y) \subset M$.

(The equalites noted in parantheses follow directly from the equality $M_{e}=M_{s e}$; see Remark 10.)

The above 'Theorem III is a generalization of the retract theorem given by $T$. Wazewski in [37]; see also [22] for a generalized form.

As a trivial consequence of Theorem III, we obtain the following

Theorem IV. Suppose that $\left(X, T^{0}, \pi\right)$ is a GSD-system, $M \subset X$, $M \neq \varnothing, M$ is open, $M_{e}=M_{s e}, S \subset M \cup M_{e} \cup M^{\prime}$ is non-empty, $S \cap\left(M_{e} \cup M^{\prime}\right)$ is a retract of $M_{e} \cup\left(M^{\prime} \cap S\right)$ but there are no retractions $S \rightarrow S \cap\left(M_{e} \cup M^{\prime}\right)$. 
Then there exists a $y \in S \cap M$, such that $\pi(y) \subset M$.

As a corollary, we have the following:

Theorex IV'. If $\left(X, T^{\mathbf{0}}, \pi\right)$ is a $G S D$-system, $M \subset X$ is open, $M \neq \emptyset$, $S$ is non-empty and $S \subset M \cup M_{e}, M_{e}=M_{s e}$, there is a retraction $M_{e} \rightarrow S \cap M_{e}$,

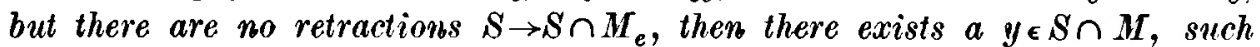
that $\pi(y) \subset M$.

Another simple consequence of Theorem III is the following

THeorem V. Suppose that $\left(X, T^{0}, \pi\right)$ is a GSD-system, $M \subset X$ is non-empty, $M$ is open, $M_{e}=M_{s e}, S \subset M \cup M^{\prime}$ is non-empty and such that $S \cap M^{*}$ is a retract of $M_{e} \cup\left(M^{\prime} \cap S\right)$, but there are no vetractions $S \rightarrow S \cap M^{\prime}$. Then there exists a $y \in S \cap M$, such that $\pi(y) \subset M$.

We shall give below a modification of Theorem IV' based on Remark 7 which is a generalization of the retract theorem in the form given by Bhatia and Szegö in [6], namely the following

TheoreM IV BIS. Let $\left(X, T^{0}, \pi\right)$ be a GSD-system and let $M, N \subset X$ be two open and non-empty sets, such that $N$ is invariant and $M \subset N$. Suppose that if $y \in \partial M \cap N$ and there is an element $z \in M \cap \pi^{-1}(y)$ such that $[z, y) \subset M$, then there exists $\lambda \in T^{0}, \lambda>0$, such that $\left(y, \pi_{\lambda}(y)\right) \neq \emptyset$ and $\left(y, \pi_{\eta}(y)\right) \cap \bar{M}=\emptyset$ for every $\eta \epsilon T^{0}, \eta<\lambda$. Suppose finally that $S \subset M \cup M_{e}$ is non-empty and $S \cap M_{e}$ is a retract of $M_{e}$, but $S \cap M_{e}$ is not a retract of $S$. Under the above assumptions, there exists $y \in S \cap M$ such that $\pi(y) \subset M$.

Finally, we will formulate explicitly a very special case of Theoren III, because it will be useful for some applications in the theory of partial differential equations of the first order. Namely, we have the following

Tilforem $\mathrm{R}$. Let us consider $R_{*}=[0, \infty)$ with the natural topology and ordered semigroup structure with respect to the usual addition and the usual ordering relation $\leqslant$. Let $a>0$ be fixed and let $X$ be a topological space, $\pi:[0, a) \times X \rightarrow X$ be a mapping belonging to $\mathscr{G}([0, a)$ ) (in the sense of Definition 2 for $T=R_{*}, T^{0}=[0, a)$ ). Let $M \subset X$ be non-empty and open, such that $M_{e}=M_{s e}$, and let $S \subset M \cup M^{*}$ be non-empty, such that $S \cap M^{*}$ is a retract of $\left(S \cap M^{*}\right) \cup M_{e}$, but there are no retractions $S \rightarrow S \cap M^{*}$.

Then, there exists a $y \in \mathbb{S} \cap M$, such that $\pi(y) \subset M$.

The reduction of Theorem $R$ to Theorem III is trivial, because of the obvious fact that $T=R_{*}$ and $T^{0}=[0, a)$ fulfill all assumptions introduced in the two first sections.

\section{EQUIVALENCE OF THEOREMS III AND IV' FOR COMPACT SETS}

Suppose that $(T, \leqslant,+)$ is an abelian ordered and dense semigroup, with the neutral element 0 , which is also a minimal element and suppose that for every $T^{\prime} \subset T, T^{\prime} \neq \varnothing$, there exists inf $T^{\prime}$. Then $(T, \leqslant, T \times T,+)$ 
is an LAO-semigroup. Let $w^{0} \epsilon T, w^{0}>0$ be fixed. Then, for $T^{0}=\left[0, w^{0}\right)$ the system ' $\left.T^{0}, \leqslant, \mathscr{N}^{0},+\right)$, where $\mathscr{N}^{0}=\left\{\langle s, t\rangle \epsilon T^{0} \times T^{0}: s+t \epsilon T^{0}\right\}$ is an LAO-semigroup.

Now we assume the following condition of a local (in $T^{0}$ ) subtraction:

(S) For every $s, t \in T^{0}$ such that $s \leqslant t$ there exists exactly one element $u$ $=u(s, t) \in T^{0}$ such that $s+u=t$. We shall write $t-s \stackrel{\text { df }}{=} u(s, t)$.

THEOREM VI. Under the above assumptions on $T$ and $T^{0}$, Theorems III and IV' are equivalent for every $M$ which is relatively compact (that is such that $\bar{M}$ is compact) and fulfils the condition: $\partial M=M^{*}=M_{s e} \cup M^{\prime}$.

Proof. The implication Theorem III $\Rightarrow$ Theoren IV' is trivial bocause Theorem $I V^{\prime}$ is a special case of Theorem IV, which is of course a special case of Theorem III (without any supplementary assumptions on $T$ and $M$ ).

In order to prove the inverse implication we shall construct for a given system $\left(X, T^{\mathbf{0}}, \pi\right)$ with $T^{\mathbf{0}}$ as above and for given $M \subset X, S \subset M$ $\cup\left(M_{e} \cup M^{\prime}\right)$ another system $\left(\tilde{X}, T^{0}, \tilde{\pi}\right)$ and sets $\tilde{M} \subset \tilde{X}, \tilde{S} \subset \tilde{M} \cup \tilde{M}_{e} \cup \tilde{M}^{\prime}$, such that the set (closure of $\tilde{M})^{s}$ is empty, and then also $\tilde{M}^{\prime} \neq \varnothing$. More precisely, we shall prove the following

Proposition 2. Let $X$ be a topological space and let $\left(X, T^{0}, \tau\right)$ be a GSD-system, with $T^{\mathbf{0}}$ as above (that is $T^{0}=\left[0, w^{0}\right) \subset T, T^{0}$ fulfilling $(S))$ and $\pi \in \mathscr{G}\left(T^{0}\right)$. Let $M \subset X$ be an open set, $M \neq \emptyset, \bar{M}$ is compact, $M_{c}=M_{s e}$. Suppose that $S \subset M \cup M_{c} \cup M^{\prime}$ is non-empty such that there is a retraction $\boldsymbol{H}_{c} \cup \boldsymbol{M} \boldsymbol{I}^{\prime} \rightarrow S \cap\left(M_{e} \cup \boldsymbol{M}^{\prime}\right)$, but there are no retractions $S \rightarrow S \cap\left(\boldsymbol{M}_{e} \cup \boldsymbol{M}^{\prime}\right)$.

Then there exists a GSD-system $\left(\tilde{X}, T^{0}, \tilde{\pi}\right)$ and there are sets $\bar{H} \subset \tilde{X}$ and $\tilde{S} \subset \tilde{M} \cup \tilde{M}_{e}$, such that $\tilde{M}, \tilde{S} \neq \varnothing, \tilde{M}$ is open, $\tilde{M}_{e}=\tilde{M}_{\text {se }}$ and there is a retraction $\tilde{M}_{e} \rightarrow \tilde{S} \cap \tilde{M}_{e}$, but there are no retractions $\tilde{S} \rightarrow \tilde{S} \cap \tilde{M}_{e}$, (closure of $\tilde{M})^{s}=\emptyset$, and furthermore the following two conditions $(\alpha)$ and $(\tilde{\alpha})$ are equivalent:

(a) there is $y \in S \cap M$, such that $\pi(y) \subset M$,

(i) there is $\tilde{y} \in \bar{S} \cap \tilde{M}$, such that $\tilde{\pi}(\tilde{y}) \in \bar{M}$.

It is clear that if the above Proposition 2 holds true, then for $T$ and $M$ as we have assumed, Theorem IV' implies Theorem III.

Indeed, assuming Theorem IV' and the assumptions of Theorem III, we can apply Theorem $I V^{\prime}$ to $\left(\tilde{X}, T^{0}, \tilde{\pi}\right)$ and corresponding sets $\tilde{M}$ and $\tilde{S}$, and then we will obtain condition $(\tilde{\alpha})$. In virtue of Proposition 2, condition $(\tilde{\alpha})$ implies $(\alpha)$, and then Theorem III holds true.

Thus, under all assumptions of Theorem III and the conditions introduced above for $T$ and $T^{0}$ (in particular (S)) we will construct a system 
$\left(\tilde{X}, T^{0}, \tilde{\pi}\right)$ having the required properties. Of course $M$ is assumed to be relatively compact and such that $\partial M=M^{*}=M_{s e} \cup M^{\prime}$. Consider the set

$$
U=\left(0, w^{0}\right) \times\{0\} \cup\{0\} \times\left[0, w^{0}\right)
$$

and define an ordering relation -3 in $U$, as follows:

$$
\begin{array}{ll}
\langle t, 0\rangle-3\langle 0,0\rangle-3\langle 0, t\rangle & \text { for every } t \epsilon\left[0, w^{0}\right), \\
\langle t, 0\rangle-3\langle s, 0\rangle & \text { if and only if } \left.t \leqslant s \text { in } T \text { (in } T^{0}\right), \\
\langle 0, t\rangle-3\langle 0, s\rangle & \text { if and only if } t \leqslant s \text { in } T \text { (in } T^{0} \text { ). }
\end{array}
$$

An easy proof that this is really an ordering relation will be omitted.

Observe that $(U,-3)$ is such that for every $V \subset U, V \neq \varnothing$, such that there is a $\left\langle s_{1}, s_{2}\right\rangle \epsilon U$, for which $\left\langle s_{1}, s_{2}\right\rangle-3\left\langle t_{1}, t_{2}\right\rangle$ for every $\left\langle t_{1}, t_{2}\right\rangle \in V$. there exists inf $V$.

Moreover, the space $(U,-3)$ is also dense. There are no maximal and minimal elements in $U$.

It is clear, that the mapping

$$
T^{0} \ni t \mapsto\langle 0, t\rangle \epsilon U^{+} \stackrel{\text { df }}{=}\{0\} \times T^{0}
$$

is an increasing bijection between $\left(T^{0}, \leqslant\right)$ and $\left(U^{+}, \Im^{+}\right)$, where $\Im^{+}$ is the restriction of -3 to $U^{+}$.

Hence, the mapping

$$
T^{0} \ni t \mapsto\langle 0, t\rangle \in U
$$

can be considered as an embedding (inclusion) compatible with the ordering relation. This mapping is also a homomorphism of the (local) semigroup $T^{0}$ into the (local) semigroup $U$ in a sense which we will define below.

Let now $\mathscr{H} \subset U \times U$ be defined as follows

$$
\begin{aligned}
\mathscr{M}= & \left\{\langle\langle 0, s\rangle,\langle t, 0\rangle\rangle: s, t \in T^{0}\right\} \cup\left\{\langle\langle s, 0\rangle,\langle 0, t\rangle\rangle: s, t \in T^{0}\right\} \\
& \cup\left\{\langle\langle s, 0\rangle,\langle t, 0\rangle\rangle: s, t \in T^{0}, s+t \epsilon T^{0}\right\} \\
& \cup\left\{\langle\langle 0, s\rangle,\langle 0, t\rangle\rangle: s, t \in T^{0}, s+t \epsilon T^{0}\right\} .
\end{aligned}
$$

We now define $+^{\bullet}: \mathscr{M} \rightarrow U$ as follows:

$$
\begin{gathered}
\langle 0, s\rangle++^{\cdot}\langle 0, t\rangle=\langle 0, s+t\rangle, \quad\langle s, 0\rangle+\cdot\langle t, 0\rangle=\langle s+t, 0\rangle, \\
\langle 0, s\rangle+\cdot\langle t, 0\rangle= \begin{cases}\langle 0, s-t\rangle & \text { if } t \leqslant s \\
\langle t-s, 0\rangle & \text { if } s \leqslant t .\end{cases} \\
\langle s, 0\rangle+\cdot\langle 0, t\rangle=\langle 0, t\rangle+\langle s, 0\rangle .
\end{gathered}
$$


Here, + and - denote the addition and the subtraction (in the sense of $(S))$ in $T^{0}$.

It is easy to see that the restriction of $+^{\cdot}$ to $U^{+}$(which we will denote by the same symbol $\left.+^{-}\right)$makes $\left(U^{+},-^{+}, \mathscr{M} \cap\left(U^{+} \times U^{+}\right),++^{-}\right)$an LAOsemigroup which can be identified with $\left(T^{0}, \leqslant, \mathscr{N}^{0},+\right)$ by the mapping (16). More precisely, the mapping (16) is a bijection compatible with both the additions and both the orders. The spaces $\left(T^{0}, \leqslant\right)$ and $\left(U^{+}, 3^{+}\right)$are also topologically equivalent (by (16)), with respect to the topologies given by the ordering relations. These facts permit us to identify $\langle 0, t\rangle \epsilon U^{+}$ with $t \in T^{0}$, $+^{-}$with + , and $-^{+}$with $\leqslant$. In order to simplify, in this line, the notation with respect to the whole $U$, we will use the following convention: if $\langle s, t\rangle \in U$, then by $-\langle s, t\rangle$ we will denote the element $\langle t, s\rangle$ belonging of course also to $U$. Hence $-(-\langle s, t\rangle)=\langle s, t\rangle$. According to this convention and to the identification of $\langle 0, t\rangle$ with $t$ (for $t \in T^{0}$ ), we will write $t$ in the place of $\langle 0, t\rangle$ and $-t$ in the place of $\langle t, 0\rangle$, for every $t \epsilon T^{0}$. Then, the following formal rule: $-(-t)=t,-0=0$, for every $t \epsilon T^{0}$, is a natural consequence of the previous conventions. Moreover, we will write $\leqslant$ in the place of -3 and + in the place of + . Thus, we have $-t \leqslant 0 \leqslant s$ for every $s, t \epsilon T^{0}$, and we can write simply $U=\left(-w^{0}, 0\right] \cup$ $\cup\left[0, w^{0}\right)=\left(-w^{0}, w^{0}\right)$. This means that we can consider $(U, \leqslant)$ as an extension of $\left(T^{0}, \leqslant\right)$; moreover, the local abelian ordered group $(U, \leqslant, \mathscr{M},+)$ is in this sense an extension of the LAO-semigroup $\left(T^{0}, \leqslant, \mathscr{M}^{0},+\right)$. We have: $t \leqslant 0 \Leftrightarrow 0 \leqslant-t$ and $-t \leqslant 0 \Leftrightarrow 0 \leqslant t$ for every $t \epsilon U$ and $t \leqslant s \leqslant 0 \Leftrightarrow 0 \leqslant-s \leqslant-t$ for every $s, t \in U$. The formulas in the definition of $+^{\cdot}$ (denoted in the sequel by + ) have now the following forms:

$$
\begin{aligned}
& s+{ }^{\cdot} t=s+t \quad \text { (in the sense of }+ \text { in } T^{0} \text { for } 0 \leqslant s, t, \\
& s+{ }^{\cdot} t=-((-s)+(-t)) \quad \text { for } s, t \leqslant 0, \\
& s+{ }^{\cdot} t=\max (s,(-t))-\min (s,(-t)) \quad \text { for } 0 \leqslant s, t \leqslant 0, \\
& s+\cdot t=\max (t,(-s))-\min (t,(-s)) \quad \text { for } 0 \leqslant t, s \leqslant 0 .
\end{aligned}
$$

We have indeed in $U$ the structure of a local ordered abelian (and dense) group: $t+s=s+t, t+0=t, t+(-t)=0, t+(s+u)=(t+s)+u$ (if at least one side is well defined) and if $s, t, u \in U, u \leqslant t, 0 \leqslant s$, then $s+u \leqslant s+t$.

Furthermore, for $s, t \in U$ such that $0 \leqslant s<t$, there exists $\lambda \in U$, $\lambda>0$, such that $s+\lambda \leqslant t$ and for $s, t \in U$, such that $t<s \leqslant 0$ there exists $\lambda \in U, \lambda<0$, such that $t<s+\lambda$; this follows directly from condition (*) in Section 2.

In $U$ we will consider the topology given by the ordering relation. 
Now, we will define a space $\tilde{X}$, which is a subspace of $U \times X$, as follows:

$\langle s, y\rangle \epsilon \tilde{X}$ if and only if, there exists $x \in X$, such that $y=\pi_{\max (0, s)}(x)$. Thus, $\langle s, y\rangle \epsilon \tilde{X}$ if and only if $s \leqslant 0$ or $0 \leqslant s$ and there is $x \in X$, such that $y=\pi_{s}(x)$.

In $U \times X$ we consider the topology of the eartesian product, induced on the usual way by the topologies of $U$ and $X$. In $\tilde{X}$ we consider the restriction of the topology of $U \times X$.

Let us consider a mapping $\tilde{\pi}: T^{\mathbf{0}} \times \tilde{X} \rightarrow \tilde{X}$ defined as follows : $\tilde{\pi}(t,\langle s, y\rangle)$ $\stackrel{\mathrm{df}}{=}\left\langle t+s, \pi_{\max (0, t+s)}(x)\right\rangle$, for $t \epsilon T^{0},\langle s, y\rangle \in \tilde{X}$, where $x \in X$ is such that $y=\pi_{\max (0, s)}(x)$. This definition is correct, since for $x, x^{\prime} \in X$ such that $\pi_{\max (0, s)}(x)=\pi_{\max (0, s)}\left(x^{\prime}\right)$, we have:

$1^{0}$ if $s \leqslant 0$, then $x=x^{\prime}$ and then of course $\pi_{\max (0, s+t)}(x)=\pi_{\max (0, s+t)}\left(x^{\prime}\right)$;

$2^{0}$ if $0 \leqslant s$, then $\max (0, s)=s, \max (0, t+s)=t+s$ and then

$$
\begin{aligned}
\pi_{\max (0, t+s)}(x) & =\pi_{t+s}(x)=\pi_{t}\left(\pi_{s}(x)\right)=\pi_{t}\left(\pi_{\max (0, s)}(x)\right)=\pi_{i}\left(\pi_{\max (0, s)}\left(x^{\prime}\right)\right) \\
& =\pi_{t}\left(\pi_{s}\left(x^{\prime}\right)\right)=\pi_{t+s}\left(x^{\prime}\right)=\pi_{\max (0, t+s)}\left(x^{\prime}\right) .
\end{aligned}
$$

This means, that $\pi_{\max (0, s+l)}(x)$ does not depend on $x$, chosen in such a way that $\pi_{\max (0, s)}(x)=y$.

A simple computation gives the following equality:

$$
\begin{array}{ll}
\tilde{\pi}(u, \tilde{\pi}(t,\langle s, y\rangle))=\tilde{\pi}(u+t,\langle s, y\rangle) & \text { for } u, t \epsilon T^{0},\langle s, y\rangle \epsilon \tilde{X}, \\
& \text { such that }\langle u, t\rangle \epsilon \mathscr{M} .
\end{array}
$$

Since the continuity of $\tilde{\pi}$ follows directly from the continuity of $\pi$, we can consider $\left(\tilde{X}, T^{0}, \tilde{\pi}\right)$ as a GSD-system.

Let us define now the sets $\tilde{M}$ and $\tilde{S}$ as follows:

$$
\begin{aligned}
& \tilde{M}=\{\langle s, x\rangle \epsilon \tilde{X}: s<0\} \cup \\
& \cup\left\{\langle s, x\rangle \in X: 0 \leqslant s \text { and there is } y \in M \text { such that } x=\pi_{s}(y)\right. \\
& \text { and } \left.\pi_{u}(y) \in M \text { for every } 0 \leqslant u \leqslant s\right\},
\end{aligned}
$$

We will prove that

$$
\begin{gathered}
\text { (closure of } \tilde{M})^{s}=\emptyset, \\
\tilde{M}_{e}=\tilde{M}_{s e}, \\
\tilde{S} \cap\left(\tilde{M}_{e} \cap \tilde{M}^{\prime}\right)=\tilde{S} \cap \tilde{M}_{e}=\{0\} \times\left(S \cap\left(M_{e} \cup M^{\prime}\right)\right), \\
\text { there is a retraction } \tilde{M}_{e} \rightarrow \tilde{S} \cap \tilde{M}_{e}, \\
\text { there are no retractions } \tilde{S} \rightarrow \tilde{S} \cap \tilde{M}_{e} .
\end{gathered}
$$

In order to show (22) observe that for every $\langle s, x\rangle \epsilon \tilde{M}$, we have 
(a) $s \leqslant 0$ or (b) $0<s$ and there exists a $y \in M$ such that $x=\pi_{s}(y)$ and $\pi_{u}(y) \in M$ for $0 \leqslant u \leqslant s$. In case (a), we can find $t<s$, such that putting $u=(-t)-(-s)$, we obtain $\tilde{\pi}_{u}(\langle t, x\rangle)=\left\langle t+u, \pi_{\max (0, t+s)}(x)\right\rangle=\left\langle s, \pi_{0}(x)\right\rangle$ $=\langle s, x\rangle$.

Remark 11. It is possible to reason another way, based on the extension of $(*)$ for $U$, namely : we can find $\lambda<0$ such that $-w^{0}<\lambda+s<s \leqslant 0$ and then putting $\eta=-\lambda$, we obtain $\eta \in T^{0}$ such that

$$
\begin{aligned}
\tilde{\pi}_{\eta}(\langle\lambda+s, x\rangle) & =\left\langle\lambda+s+\eta, \pi_{\max (0, \lambda+s+\eta)}(x)\right\rangle=\left\langle s, \pi_{\max (0, s)}(x)\right\rangle \\
& =\left\langle s, \pi_{0}(x)\right\rangle=\langle s, x\rangle .
\end{aligned}
$$

In case (b) we have directly from the definition of $\tilde{M}$ an element $y \in M$ such that $x=\pi_{s}(y)$, and then we have an element $\langle 0, y\rangle \in \tilde{M}$, such that $\tilde{\pi}_{s}(\langle 0, y\rangle)=\langle s, x\rangle$. Then, each element $\langle s, x\rangle$ of $\tilde{M}$ is an image by $\tilde{\pi}_{u}$ of some element $\langle t, y\rangle \in M$, for some $u \epsilon T^{0}$.

If $\langle s, x\rangle \epsilon \partial \tilde{M}$, then (c) $s=0$ (and we can use the same reasoning as in case (a) for $\langle s: x\rangle \in \tilde{M}$ ), or (d) $0<s$. In this case (d), there is a sequence $\left\{\left\langle s_{n}, x_{n}\right\rangle\right\}_{n=1,2, \ldots} \subset \tilde{M}$ convergent to $\langle s, x\rangle$. For every $n$, there is a $y_{n} \in M \subset \bar{M}$, such that $\pi\left(s_{n}, y_{n}\right)=x_{n}$.

Since $M$ is relatively compact, we can choose a subsequence $\left\{y_{a_{n}}\right\}$ of $\left\{y_{n}\right\}$, convergent to a point $y \in \bar{M}$; the subsequence $\left\{s_{a_{n}}\right\}$ is obviously convergent to $s$. Hence, in virt ue of the continuity of $\pi$, we have: $\pi(s, y)=x$.

Then, in virtue of the obvious relation $\langle 0, y\rangle \in($ closure of $\tilde{M})$, we have: for every $\langle s, x\rangle \in \partial \tilde{M}$ such that $s>0$, there is an element $\langle 0, y\rangle$ belonging to the closure of $\tilde{M}$, such that $\tilde{\pi}(s,\langle 0, y\rangle)=\langle s, x\rangle$. The proof of (22) is completed.

Now we will prove (23). Let $\langle s, x\rangle$ be a point of $\tilde{M}_{e}$. This means that (e) $s=0$ and $x \epsilon \partial M=M^{*}$, or (f) $0<s, x \in \partial M$ and there is a point $y \in M$, such that $\pi_{s}(y)=x, \pi_{u}(y) \in M$ for $0 \leqslant u<s$. In case (e), we have $\langle s, x\rangle \in M_{s e}$, because $\tilde{\pi}_{\eta}(\langle s, x\rangle)=\tilde{\pi}_{\eta}(\langle 0, x\rangle)$ does not belong to the closure of $\tilde{M}$, for every $\eta>0, \eta \leqslant \lambda$ with some $\lambda>0$; this follows directly from the fact, that $x \in M^{*}$.

In case (f), $x \in M_{e}=M_{s e}$ and then also $\langle s, x\rangle \in \tilde{M}_{s e}$.

We will prove (24). In virtue of (22) the set $\tilde{M}^{\prime}$ is empty and then $\tilde{S} \cap\left(\tilde{M}_{e} \cup \tilde{M}^{\prime}\right)=\tilde{S} \cap \tilde{M}_{e}$. Furthermore, $\langle s, x\rangle \epsilon \tilde{S}$ if and only if $s=0$, then $\langle s, x\rangle \in \tilde{S} \cap \tilde{M}_{e}$ if and only if $s=0$ and $x \in S \cap \partial M=S \cap\left(M_{e} \cup M^{\prime}\right)$.

In order to prove $(25)$ consider the mapping

$$
\varphi: \tilde{M}_{e}{ }^{3}\langle s, x\rangle \mapsto\langle 0, x\rangle \epsilon \tilde{S} \cap \tilde{M}_{e}
$$

which is continuous and fulfills of course the condition $\left.\varphi\right|_{\tilde{S} \cap \tilde{M}_{e}}=\operatorname{id}{\tilde{S} \cap \tilde{M}_{e}}_{e}$, and is therefore a retraction. 
Finally, suppose that there is a retraction $\tilde{\psi}: \tilde{S} \rightarrow \tilde{S} \cap \tilde{M}$.

The mapping $\tilde{\psi}$ induces in a natural way a mapping $\psi: S \rightarrow S \cap \partial M$ $=S \cap\left(M_{c} \cup M^{\prime}\right)$ as follows: $\psi(x)$ is equal to that element $y \in \partial M$ for which the pair $\langle 0, y\rangle$ is the value of the mapping $\tilde{\psi}$ at the point $\langle 0, x\rangle \epsilon \tilde{S}$. The mapping $w$ is continuous and its restriction to $S \cap\left(M_{e} \cup M^{\prime}\right)$ is the identity, because the restriction of $\tilde{\psi}$ to the set $\tilde{S} \cap \tilde{M}_{e}$ is the identity. Hence $\psi$ is a retraction $S \rightarrow S \cap\left(M_{e} \cup M^{\prime}\right)$; the existence of such a retraction is, however, impossible in virtue of the assumptions of Theorem III supposed here. This proves (26).

In order to finish the proof of Proposition 2 (and then also the proof of Theorem VI) we shall show the equivalence $(\alpha) \Leftrightarrow(\tilde{\alpha})$. Suppose that $(\tilde{\alpha})$ is fulfilled. Then, there is a $\langle s, x\rangle \epsilon \tilde{M}$ such that $\tilde{\pi}_{u}(\langle s, x\rangle) \epsilon M$ for every $u \in T^{0}$. This means that there is $y \in M$ such that $\pi_{s}(y)=x$ and $\pi_{u}(y) \in M$ for $0 \leqslant u \leqslant s$ and, moreover, $\pi_{s+u}(y) \in M$ for every $u \in T^{0}$ such that $s+u \epsilon T^{0}$. Let $t \epsilon T^{0}$ be arbitrary fixed. If $t \leqslant s$, then of course $\pi_{t}(y) \epsilon M$. If $s<t$, then putting $u=t-s$, we obtain $\pi_{t}(y)=\pi_{u}\left(\pi_{s}(y)\right)=\pi_{u+s}(y) \epsilon M$, since $u+s=t \epsilon T^{0}$. This means that $(\alpha)$ is fulfilled.

Conversely, if $(\alpha)$ is satisfied, then $(\tilde{\alpha})$ is also satisfied, since for $\langle 0, y\rangle \epsilon \tilde{M}$, with $y \in M$ such that $\pi(y) \subset M$ we have obviously $\tilde{\pi}_{s}(\langle 0, y\rangle) \epsilon \tilde{M}$ for every $s \in T^{0}$.

Remark 12. It was sufficient to prove only the implication $(\tilde{\alpha}) \Rightarrow(\alpha)$, but since the converse implication is immediate, we formulated the corresponding condition in the form of an equivalence.

Remark 13. All applications givan in the second part of the present paper concern questions in which corresponding sets $M$ are relatively compact, the space $T$ is cqual to $[0, \infty)$ and the LAO-semigroup $T^{0}$ is equal to a bounded interval $\left[0, w^{0}\right)$; this moans that all assumptions of Theorem VI are fulfilled in these particular problems. We underline this fact, because in the papers [22]-[25] the retract theorem in the version of 'Theorem $I V^{\prime}$ is presented and applied.

In virtue of the above remark, this form is enough to the applications in the theory of partial differential equations. Of course, having here the general form (Theorem III) we will use it in the sequel. Note finally, that the reasoning presented in the present section in the proof of the last theorem, was used by the author previously in a special case in [25].

Remark 14. From the formal point of view, the equivalence of Theorems III and IV' is trivial, since the both are true; the essence of Theorem VI is contained in the fact that in the case of compact sets $M$ such that $\partial M=M^{*}=M_{s e} \cup M^{\prime}$ and $T^{0}$ having property (S), the formally more general Theorem III gives really nothing new with respect to Theorem IV'. 


\section{PART II}

\section{INVESTIGATIONS OF LOCAL BEHAVIOR OF SOLUTIONS OF SYSTEMS OF ORDINARY DIFFERENTIAL EQUATIONS}

We will give an example of applications of Theorem III (more precisely, of a special case of Theorem III, namely Theorem R) from Section 8 of Part I, showing that the generalizations of the classical retract theorem for GSD-systems are useful - especially from the technical point of view even in the theory of ordinary differential equations. For further comments we refer to Section 1.4.

1.1. Notation and assumptions. Let $a$ be a positive real number and let $f: U \rightarrow R^{n}$, where $U$ is an open subset of $R^{n}$ be a lipschitzian (vector-) function, such that for every $y^{0} \epsilon R^{n}$, there exists a solution (necessarily at most one) $y$ of the following Cauchy problem

$$
\begin{aligned}
& y^{\prime}=f(y), \\
& y(0)=y^{0},
\end{aligned}
$$

defined in $[0, a)$. Of course, in virtue of the assumptions of regularity of $f$ (the Lipschitz condition), the local existence of solutions is trivial; the only non-trivial assumption in this situation, is that every solution exists in $[0, a)$.

The unique solution of (1.1)-(1.2) we denote by $y\left[y^{0}\right]$; thus we have:

$$
\frac{d}{d t} y\left[y^{0}\right](t)=f\left(y\left[y^{0}\right](t)\right) \quad \text { for } t \in[0, a) \text { and } y\left[y^{0}\right](0)=y^{0} \text {. }
$$

1.2. THEOREM 1. Assume the above conditions. Assume, moreover, that $r$ is a positive number and that for every $y \in R^{n}$ belonging to the sphere

$$
S(r)=\left\{u \epsilon R^{n}: \sum_{i=1}^{n} u_{i}^{2}=r^{2}\right\}
$$

(which is assumed to be contained in $U$ ) the following inequality

$$
\sum_{i=1}^{n} y_{i} f_{i}\left(y_{1}, \ldots, y_{n}\right)>0
$$

holds true. ball

Under the above assumptions, there exists $y^{0} \in R^{n}$, belonging to the open

$$
B(r)=\left\{u \epsilon R^{n}: \sum_{i=1}^{n} u_{i}^{2}<r^{2}\right\}
$$


such that the solution $y\left[y^{0}\right]$ of (1.1)-(1.2) fulfills the inequality

$$
\sum_{i=1}^{n}\left(y_{i}\left[y^{0}\right](t)\right)^{2}<r
$$

for every $t \in[0, a)$. This means, that $y\left[y^{0}\right](t)$ belongs to the ball $B(v)$ for every $t \in[0, a)$.

1.3. Proof. Let us consider $X=R^{n}, M=B(r), S=\bar{M}(=B(r) \cup S(r))$, $T=[0, \infty), T^{0}=[0, a)$. Define $\pi: T^{0} \times X \rightarrow X$ in such a way that $\pi(t, x)$ $\stackrel{d f}{=} y[x](t)$. Since $f$ does not depend on $t$, we have obviously: $\pi(s, \pi(t, x))$ $=\pi(s, y[x](t))=y[y[x](t)](s)=y[x](t+s)=\pi(t+s, x)$, for every $s, t \geqslant 0$, such that $t+s<a$. The mapping $\pi$ is continuous in virtue of wellknown theorems from the theory of differential equations. Hence $\left(X, T^{0}, \pi\right)$ forms a GSD-system. The set $M$ is open and non-vide. The boundary $\partial M$ of $M$ is equal to $M^{\prime} \cup M_{e}$. Since $M_{e}=M_{s e}$ (this is a simple consequence of inequality (1.4) for $y \epsilon S(r)=\partial M)$, we have: $\partial M=M^{*}$ $=M^{\prime} \cup M_{e}=M^{\prime} \cup M_{s e}$. Of course the set $S$ is equal to $M \cup M^{*}$ and then $S \cap M^{*}=S \cap\left(M^{\prime} \cup M_{e}\right)=M^{*}=\left(M^{\prime} \cup M_{e}\right)$ is not a retract of $S$, since there are no retractions of the closed ball $\overline{B(r)}$ on his boundary $S(r)$. On the other hand $S \cap M^{*}=\partial M$ is a trivial retraction of $\left(S \cap M^{*}\right) \cup M_{e}$ $=M^{*} \cup M_{e}=\partial M$.

Then all assumptions of Theorem $\mathbf{R}$ from Section 8 of Part $I$, are fulfilled for the above $X, T, M, S$ and $\pi$, and then the assertion of this theorem holds true. This means that there exists $x \in M$, such that $\pi(x) \subset M$. The last inclusion is equivalent to the relation $\pi(t, x)=y[x](t) \in M$ for $t \in[0, a)$. Hence, there is a solution $y[x]$ (for some $x \in B(r)$ ) of $(1.1)-(1.2)$ with $y^{0}$ replaced by $x$, satisfying $(1.5)$ for $t \epsilon[0, a)$.

\subsection{Remarks.}

$1^{\circ}$ In virtue of Remark 1a from the first part (Section 1 ), it is possible put $T^{0}=T$. This means that also a global version of Theorem 1 can be deduced from Theorem $R$.

$2^{\circ}$ The above Theorem 1, a local version of the classical retract theorem of T. Ważewski, seems to give no essentially new results; it is only a modification of well-known results, a slight formal generalization of the original version [37], [38], [41]. We have presented it here to illustrate some technical advantages of using local forms of extended retract theorems stated for GSD-systems. Notice also that semigroup structure introduced in Part I instead of the natural group structure in $R$ considered in the classical versions of the Ważewski's retract theorem is very useful and makes it possible to state several results in more general form. This is important in the case of time-lag differential equations; see for instance [26]. 
The material advantages of generalizing the retract theorem to GSDsystems can be observed in the applications of the main results from Part I to partial differential equations. Examples are presented below.

$3^{0}$.The formally more general case of equations $u^{\prime}=h(t, u)$ with $h:[a, b) \times R^{k} \rightarrow R^{k} \quad(a \geqslant 0)$ fulfilling suitable assumptions (we omit the details), can be reduced to the case discussed above, by using the usual method: $n=k+1, \quad U=[a, b) \times R^{k} \subset R^{n}, \quad y_{1}=t, y_{i+1}=u_{i}, f_{i+1}(y)=$ $=h_{i}(t, u)(i=1, \ldots, n-1), f_{1}(y)=1$.

\section{PRELIMINARIES NECESSARY FOR A GENERALIZED RETRACT THEOREM FOR FAMILIES OF SURFACES, WITH APPLICATIONS TO PARTIAL DIFFERENTIAL EQUATIONS}

2.1. Notation. We shall use the following notation: $R=(-\infty,+\infty)$ is - as usual - the real line, $R_{+}=(0,+\infty), R_{*}=[0,+\infty)$. The usual notation for closed, open and "semiclosed" (of the two types) intervals will be also used: $[a, b],(a, b),(a, b],[a, b)$.

If $\Delta$ is a compact subset of $R$ or of $R^{2}=R \times R$, then by $\mathscr{C}(\Delta, R)$ we denote the Banach space of all continuous functions from $\Delta$ into $R$, provided with the usual norm: $\|\lambda\|=\max \{|\lambda(x)|: x \in \Delta\}$.

Let $A$ be a positive number and let $F$ be a real, non-negative, continuous function defined in $[0, A]$. We put

$$
\begin{aligned}
Q^{0}(A ; F) & =\left\{\langle x, y\rangle \epsilon R^{2}: 0 \leqslant x<A,|y| \leqslant F(x)\right\}, \\
Q(A ; F) & =Q^{0} \cup\{[A, \infty) \times[-F(A), F(A)]\} .
\end{aligned}
$$

In particular, if $F(x)=\delta-\gamma x$ with some non-negative constants $\delta$ and $\gamma$ such that $\gamma \cdot A \leqslant \delta$, then instead of $Q(A ; F)$ we will write $Q(A ; \delta, \gamma)$; precisely

$$
Q(A ; \delta, \gamma) \stackrel{\text { df }}{=} Q(A ;\{[0, A] \epsilon x \mapsto \delta-\gamma x \in R\})
$$

2.2. Preliminary definitions and propositions. Let $I \subset R$ be a compact interval, $K$ be a positive constant, $p$ and $q$ be two real functions defined in $I$, fulfilling the Lipschitz condition with the constant $K$ and satisfying the inequality $p(y)<q(y)$ for $y \in I$. Denote by $S(I, K, p, q)$ the set of all functions $\varphi \in \mathscr{C}(I, R)$, such that $\left|\varphi(y)-\varphi\left(y^{\prime}\right)\right| \leqslant K\left|y-y^{\prime}\right|, p(y) \leqslant \varphi(y)$ $\leqslant q(y)$ for every $y, y^{\prime} \in I$.

If $q(y)=-p(y)>0$ for $y \in I$, then instead of $S(I, K, p,-q)$, we will write simply $S(I, K, q)$; if $I=[-B, B]$ and $q(y)=D=$ const, then we put:

$$
S_{K, B, D} \stackrel{\text { df }}{=} S([-B, B], K,\{[-B, B] \ni y \mapsto D \epsilon R\}) .
$$


Definition of condirion (W). Let $S^{0}$ be a subset of $S(I, K, p, q)$, which is a subset of $\mathscr{C}(I, R)$. We say that $S^{0}$ fulfils condition (W) (shortly: $\left.S^{0} \epsilon(W)\right)$, if and only if the following set

$$
S^{0^{\prime}} \stackrel{\text { df }}{=}\left\{\varphi \in \mathbb{S}^{0}: \varphi(y)=p(y) \text { for some } y \in I \text { or } \varphi(y)=q(y)\right.
$$

for some $y \in I\}$

satisfies the condition

$$
S^{0^{\prime}} \neq \emptyset \text { and there are no retractions } S^{0} \rightarrow S^{0^{\prime}} .
$$

IV have the following obvious

Proposition 1. If $S^{0}$ is connected (in $\mathscr{C}(I, R)$ of course) and $S^{0}$ is nonempty but not connected, then $S^{0} \epsilon\left(W^{\top}\right)$.

Corollary 1. If $S^{0} \subset S(I, K, p, q)$ is comnected and $S^{0^{\prime}}=\{p, q\}$, then $S^{0} \epsilon(W)$.

Proposition 2. If $q(y)=-p(y)>0$ for $y \epsilon I$, and $S^{0} \subset S(I, K, q)$ is convex and compact in $\mathscr{C}(I, R), S^{0^{\prime}} \neq \emptyset$, then $S^{0} \epsilon(W)$.

This is a special case of well-known results from the theory of retracts. Since we wish to make this account reasonably selfcontained, we shall sketch here an easy proof, repeting the classical reasoning, based on wellknown fixed point theorems.

First of all, we observe that every continuous mapping from $S^{0}$ into itself has a fixed point; it follows directly from the properties of $S^{0}$ assumed here, in virtue of the classical fixed point theorems. Suppose now, that $r: S^{0} \rightarrow S^{0^{\prime}}$ is a retraction and consider a mapping $\varrho: S^{0} \rightarrow S^{0}$ defined by the formula: $\varrho(\chi)=-r(\lambda)$ for $\lambda \in S^{0}$. Of course $\varrho$ is continuous, and then it has a fixed point $\lambda^{0}$, belonging obviously to $S^{0^{\prime}}$. We have: $\lambda^{0}=\varrho\left(\lambda^{0}\right)=-r\left(\lambda^{0}\right)=-\lambda^{0}$, since $\lambda^{0} \epsilon S^{0^{\prime}}$. Then $\lambda^{0}=0$, but the zerofunction does not belong to $\mathrm{S}^{\circ \prime}$; we have a contradiction, so there are no retractions from $S^{0}$ onto $S^{0^{\prime}}$.

Corollary 2. The set $S_{1}=S(I, K, q)$ fulfils $(W)$, because it is compact and convex.

Corollary 3. The set $S_{2}=S_{2}(I, K, q ; \hat{K})$ of all $C^{1}$-functions $p$ belonging to $S(I, K, q)$, such that $\left|\varphi^{\prime}(y)\right| \leqslant K$ and $\left|\varphi^{\prime}(y)-\varphi^{\prime}(\bar{y})\right| \leqslant \hat{K}|y-\bar{y}|$, for $y, \bar{y} \in I$, fulfils (for every $K \in R_{*}$ ), condition (W).

\section{APPLICATIONS OF GENERAL RESULTS FOR FAMILIES OF REGULAR SURFACES}

3.1. Notation and assumptions. Suppose that $K$ and $B$ be positive constants, $p$ and $q$ be two functions belonging to $\mathscr{C}([-B, B], R)$ fulfilling the Lipschitz condition with the constant $K$, and such that $p(y)<0$ $<q(y)$ for $y \in[-B, B]$. 
Throughout the present chapter, the set $S([-B, B], K, p, q)$ will be shortly noted by $S^{*}$.

Suppose that $S^{0} \subset S^{*}$ is convex and such that

$\left\{\lambda(y): \lambda \in S^{0}\right\}=[p(y), q(y)] \quad$ for every $y \in[-B, B], S^{0^{\prime}}=\{p, q\}$.

The above conditions imply that $\mathcal{S}^{0} \epsilon(W)$ (see Proposition 1).

Let $A \in R_{+}$be fixed and let $F:[0, A] \rightarrow R_{+}$be a decreasing lipschitzian function, such that $F^{\prime}(0)=B ; F$ will be fixed through out the present chapter.

We will denote by $Q^{0}$ and $Q$, the sets $Q^{0}(A ; F)$ and $Q(A ; F)$ (see (2.2)) respectively. Let $g$ and $h$ be two functions belonging to $\mathscr{C}\left(\overline{Q^{0}}, R\right)$, such that $g(x, y)<h(x, y)$ for $\langle x, y\rangle \in Q^{0},|g(x, y)-g(\bar{x}, \bar{y})|, \mid h(x, y)-$ $-h(\bar{x}, \bar{y})\left|\leqslant L_{1}\right| x-\bar{x}\left|+L_{2}\right| y-\bar{y} \mid$ for $\langle x, y\rangle,\langle\bar{x}, \bar{y}\rangle \in Q^{0}$, and finally

$$
g(0, y)=p(y), \quad h(0, y)=q(y) \quad \text { for } y \in[-B, B] .
$$

Let $\mathscr{D}^{0}$ be a family of functions belonging to $\mathscr{C}\left(\overline{Q^{0}}, R\right)$ such that:

(i) $\quad\left\{\left.\varphi\right|_{\{\langle 0, y\rangle:|v| \leqslant B\}}: \varphi \in \mathscr{D}^{0}, p(y) \leqslant \varphi(y) \leqslant q(y)\right\}=S^{0}$;

(ii) If for $\varphi, \psi \in \mathscr{D}^{0}$ there is some $x^{0} \in[0, A)$, such that $\varphi(x, y)=\psi(x, y)$ for $0 \leqslant x \leqslant x^{0},|y| \leqslant F(x)$, then $\varphi(x, y)=\psi(x, y)$ for every $\langle x, y\rangle \epsilon Q^{0}$;

(iii) For every $\varphi \in \mathscr{D}^{0}$, every $\varepsilon \epsilon R_{+}$and every $x^{\prime} \in[0, A]$, there is a $\delta \in R_{+}$such that if $\psi \in \mathscr{D}^{0}$ and $|\varphi(0, y)-\psi(0, y)|<\delta$ for $|y| \leqslant B$, then $\left|\varphi\left(x^{\prime}, y\right)-\psi\left(x^{\prime}, y\right)\right|<\varepsilon$ for $\left|y^{\prime}\right| \leqslant F\left(x^{\prime}\right)$;

(iv) $|\varphi(x, y)-\varphi(\bar{x}, \bar{y})| \leqslant L_{1}|x-\bar{x}|+L_{2}|y-\bar{y}|$ for every $\varphi \epsilon \mathscr{D}^{0}$ $\mid$ and every $\langle x, y\rangle,\langle\bar{x}, \bar{y}\rangle \in Q^{0}$;

(v) If $\varphi \epsilon \mathscr{D}^{0}$ and $\varphi\left(x^{0}, y^{0}\right)=h\left(x^{0}, y^{0}\right)$ (resp. $\left.\varphi\left(x^{0}, y^{0}\right)=g\left(x^{0}, y^{0}\right)\right)$ for some $\left\langle x^{0}, y^{0}\right\rangle \in Q^{\mathbf{0}}$, then: $\left.F\left(x^{0}\right)\right]$,

$1^{0} \varphi\left(x^{0}, y\right) \neq g\left(x^{0}, y\right) \quad\left(\right.$ resp. $\left.\quad \varphi\left(x^{0}, y\right) \neq h\left(x^{0}, y\right)\right)$ for $y \in\left[-F\left(x^{0}\right)\right.$,

$2^{0}$ there exists a $\sigma \epsilon R_{+}$such that for every $x \in\left[x^{0}, x^{0}+\sigma\right)$ there exists a $y \in[-F(x), F(x)]$ such that $\varphi(x, y)>h(x, y)$ (resp. $\varphi(x, y)$ $<g(x, y))$.

3.2. THEOREM 2. Under the assumptions from the above Section 3.1, there exists a $\varphi \in \mathscr{D}^{0}$ such that $g(x, y)<\varphi(x, y)<h(x, y)$ for $\langle x, y\rangle \epsilon Q^{0}$.

Proof. Using the notation introduced in Chapter 2, we put for $x \in R_{*}$ :

$$
\begin{gathered}
Q_{(x)}=\{\langle x, y\rangle \in Q: y \in R\}(=Q \cap\{\langle x, y\rangle: y \in R\}=\{\langle x, y\rangle: \\
\left.\left.|y| \leqslant F(x) \text { for } x \epsilon^{\prime}[0, A),|y| \leqslant F(A) \text { for } x \geqslant A\right\}\right), \\
\left\lfloor Q_{x}=\bigcup\left\{Q_{(l)}: t \epsilon[0, x]\right\} .\right.
\end{gathered}
$$


For $\varphi^{0} \epsilon \mathscr{D}^{0}$ and $x>A$ we put

$$
\varphi(x, y) \stackrel{d f}{=} \varphi^{0}(A, y)
$$

obtaining on this way an extension $\varphi$ of the given function $\varphi^{0}$; the extended function $\varphi$ is defined and lipschitzian in $Q$.

By the same formula we extend $g$ and $h$ on the whole set $Q$; we will denote these extensions also by $g$ and $h$; so we will now consider $g$ and $h$ as functions defined on $Q$.

By $\mathscr{D}$ we denote the family of all extensions of functions belonging to $\mathscr{D}^{0}$ :

$$
\mathscr{D}=\left\{\varphi: Q \rightarrow R \mid \varphi \text { is given by (3.3) for some } \varphi^{0} \epsilon \mathscr{D}^{0}\right\} \text {. }
$$

For $\varphi \epsilon \mathscr{D}$ and $x \epsilon R_{*}$ we denote by $\varphi_{(x)}$ (resp. $\varphi_{x}$ ) the restriction of $\varphi$ to the set $Q_{(x)}$ (resp. $Q_{x}$ ). Similarly, by $g_{(x)}, g_{x}, h_{(x)}, h_{x}$ we denote the corresponding retractions of $g$ and $h$, to the sets $Q_{(x)}$ and (respectively) $Q_{x}$.

Using this notation, we can write now condition (ii) equivalently in the form:

$$
\left\{x \epsilon R_{*}, \varphi, \psi \epsilon \mathscr{D}, \varphi_{x}=\psi_{x}\right\} \Rightarrow \varphi=\psi .
$$

Put $Z=Z(\mathscr{D}) \stackrel{\text { df }}{=}\left\{\varphi_{k}: \varphi \in \mathscr{D} \cup\{g, h\}, x \in R_{*}\right\}$ and define a set $X$ $=X(\mathscr{D}) \subset Z \times R_{*}$ in such a way that

$$
\langle z, x\rangle \in X \stackrel{\text { df }}{\Leftrightarrow}\left\{\text { there exists } \varphi \in \mathscr{D} \cup\{g, h\} \text {, such that } \varphi_{x}=z\right\} \text {. }
$$

If $x \in R_{*}$ and $\lambda \in \mathscr{C}\left(Q_{x}, R\right)$, then we put $|\lambda|_{x}=\|\lambda\|$; this means that $|\lambda|_{x}=\max \left\{|\lambda(t, y)|:\langle t, y\rangle \epsilon Q_{x}\right\}$. For $\langle z, x\rangle \in X$ and $\eta, \gamma \in R_{+}$, we put

$$
N_{\eta, \gamma}(z, x) \stackrel{d f}{=}\left\{\langle u, t\rangle \in X: t \epsilon(x-\eta, x+\eta) \cap R_{*},\left|\varphi_{w}-\psi_{w}\right|_{w}<\gamma\right.
$$

for $w=\max (t, x)$, where $\varphi, \psi \in \mathscr{D} \cup\{g, h\}$ are such that $\left.\varphi_{x}=z, \psi_{t}=u\right\}$.

LEMMA 1. If $\langle z, x\rangle,\langle\bar{z}, \bar{x}\rangle \in X$ and $\eta, \gamma, \bar{\eta} \bar{\gamma} \epsilon R_{+},\langle\overline{\bar{z}}, \overline{\bar{x}}\rangle \in N_{\eta, x}(z, x)$

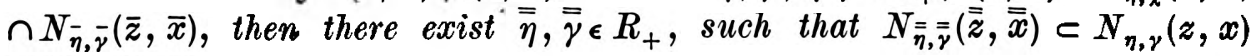
$\cap N_{\bar{\eta}, \bar{\gamma}}(\bar{z}, \bar{x})$.

We omit an elementary proof of this lemma.

CorollaRY 4. The family $\left\{N_{\eta, \mathrm{o}}(z, x): \eta, \delta \in R_{+},\langle z, x\rangle \in X\right\}$ induces in the usual way - a topology in $X$; we will denote this topology by $\mathscr{T}$.

Remark. It is easy to see that $(X, \mathscr{T})$ is a Hausdorff space; this fact, however, is inessential in the sequel.

We will consider now $T=R_{*}$ and an LAO-semigroup (in this case an abelian ordered semigroup; see Remark la in Section 1 of Part I) $(T, \leqslant, T \times T,+)$.

Now we define a mapping

$$
\pi: T \times X \rightarrow X
$$


by the following formula

$$
\pi(t,\langle z, x\rangle) \stackrel{\text { df }}{=}\left\langle\varphi_{x+t}, x+t\right\rangle,
$$

where $\varphi \in \mathscr{D}$ is such that $\varphi_{x}=z$.

This definition is correct, because of condition (ii); it is sufficient to observe, that (3.4) implies the independence of the value $\pi(t,\langle z, x\rangle)$ on the choise of $\varphi \epsilon \mathscr{D}$ in such a way that the required condition $\varphi_{x}=z$ is satisfied.

We consider $T \times X$ as a topological space, with the topology of the cartesian product given as usual by $\mathscr{T}$ and the restriction to $R_{*}=T^{r}$ of the natural topology of $R$.

\section{LeMma 2. The mapping $\pi$ is continuous.}

Proof. We use conditions (iii) and (iv). Let $\langle t,\langle z, x\rangle\rangle$ and $\eta, \delta \in R_{+}$ be fixed. Let $\varphi \in \mathscr{D}$ be such that $\varphi_{x}=z$; this means that $\pi(t,\langle z, x\rangle)$ $=\left\langle\varphi_{x+t}, x+t\right\rangle$. Consider $[x, x+t+\eta]$. Since all functions belonging to $\mathscr{D}$ satisfy the Iipschitz condition (this follows directly from (iv) and (3.3)), we can find $\bar{\eta} \epsilon R_{+}$, such that if $\psi \epsilon \mathscr{S}$ and $\left|\varphi_{x}-\psi_{x}\right|_{x}<\delta / 2$, then $\mid \psi_{(s)}(s, y)-$ $-\varphi_{(s)}(s, y) \mid<\delta$ for $s \epsilon(x-\bar{\eta}, x+\bar{\eta})$ and every $y$ such that $\langle s, y\rangle \epsilon Q$. We can find a finite sequence of points $x_{1}, \ldots, x_{p}$, such that: $1^{\circ} x_{1}<x_{2}<\ldots<x_{p}$, $2^{\circ}[x, x+t+\eta] \subset \bigcup_{i=1}^{p-1}\left(x_{i}, x_{i+1}\right), 3^{\mathbf{o}} x_{j+1}-x_{j}<\bar{\eta}$ for $j=1, \ldots, p-1$. Condition (iii) implies the following: for every $x_{j}$, there exists $\delta_{j}>0$, such that if $\psi \in \mathscr{D}$ and $|\psi(0, y)-\varphi(0, y)|<\delta_{j}$ for $|y| \leqslant B$, then $\mid \psi\left(x_{j}, y\right)-$ $-\varphi\left(x_{j}, y\right) \mid<\delta / 2$ for $y$ such that $\left\langle x_{j}, y\right\rangle \in Q$. Putting $\mu=\min \left(\delta_{1}, \ldots, \delta_{p}\right)$ and $\varrho=\eta / 2$, we obtain the following implication: $\langle\tilde{z}, \tilde{x}\rangle \in N_{\varrho, \mu}(z, x)$, $\tilde{t} \epsilon(t-\varrho, r+\varrho) \Rightarrow \pi(\tilde{t},\langle\tilde{z}, \tilde{x}\rangle) \epsilon N_{\eta, \delta}(\pi(t,\langle z, x\rangle))$, which means that $\pi$ is continuous.

Lemira 3. The set

$$
S_{a}=\left\{\left\langle g_{0}, 0\right\rangle,\left\langle h_{0}, 0\right\rangle\right\}
$$

is a retract of

$$
\begin{aligned}
S_{b}=\left\{\langle z, x\rangle \epsilon X: z_{(x)}(y)=\right. & g_{(x)}(y) \text { for some } y \in Q_{(x)} \\
& \text { or } \left.z_{(x)}(y)=h_{(x)}(y) \text { for some } y \epsilon Q_{(x)}\right\},
\end{aligned}
$$

but there are no retractions $\left(S^{0} \times\{0\}\right) \cup S_{a} \rightarrow S_{a}$.

Proof. We can identify $S_{a}$ and $S^{0} \times\{0\}$ with $S^{0^{\prime}}=\{p, q\} \quad\left(q=g_{0}\right.$, $\left.p=h_{0}\right)$ and $S^{0}$ respectively; applying Corollary 1 of Proposition 1 from Chapter 2, we obtain immodiately that there are no retractions from $\left(S^{0} \times\{0\}\right) \cup S_{a}$ onto $S_{a}$.

In order to prove the existence of a retraction $S_{b} \rightarrow S_{a}$, we observe that the mapping $\langle z, x\rangle \mapsto\left\langle g_{0}, 0\right\rangle$ (resp. $\langle z, x\rangle \mapsto\left\langle h_{0}, 0\right\rangle$ ) for $z$ such that

$$
z_{(x)}(y)=g_{(x)}(y) \quad\left(\text { resp. } z_{(x)}(y)=h_{(x)}(y)\right) \quad \text { for some } y \in Q_{(x)}
$$


is continuous, as a composition of two projections:

$$
\langle z, x\rangle \mapsto\left\langle g_{u}, u\right\rangle \quad\left(\text { resp. }\langle z, x\rangle \mapsto\left\langle h_{u}, u\right\rangle\right)
$$

for $z$ fulfilling $\left({ }^{\circ}\right)$, with $u=\min \left\{t: z(t, y)=g(t, y)\right.$ for some $\left.y \in Q_{t}\right\}$ (resp. $u=\min \left\{t: z(t, y)=h(t, y)\right.$ for some $\left.\left.y \in Q_{l}\right\}\right)$, and

$$
\left\langle g_{x}, x\right\rangle \mapsto\left\langle g_{0}, 0\right\rangle \quad\left(\text { resp. }\left\langle h_{x}, x\right\rangle \mapsto\left\langle h_{0}, 0\right\rangle\right) .
$$

The continuity of (') follows directly from ( $v)$; the continuity of. $\left({ }^{\prime \prime}\right)$ is obvious.

Put

$$
\begin{array}{r}
M \stackrel{\mathrm{df}}{=}\left\{\langle z, x\rangle: g_{x}(y)<z_{x}(y)<h_{x}(y) \text { for every } y,\right. \\
\text { such that }\langle x, y\rangle \in Q\} .
\end{array}
$$

Of course, the set $M$ is open. The set $M_{e}$ has as its elements such pairs $\langle z, x\rangle$ belonging to $X$, that $0<x<A$ and

$1^{0} z_{(v)}(y)<h_{(v)}(y)$ and $z_{(v)}(y)>g_{(v)}(y)$ for $0 \leqslant v<x$ and $y \epsilon Q_{(v)}$, and $2^{\circ}$ there is $y \in Q_{(x)}$, such that $z_{(x)}(y)=h_{(x)}(y)$ or $z_{(x)}(y)=g_{(x)}(y)$. Hence, in virtue of (v), we have: $M_{e}=M_{s e}$.

Moreover, $\langle z, x\rangle \in M^{\prime}$ if and only if $x=0$ and there is $y \in[-B, B]$ such that $z_{0}(y)=g_{0}(y)$ or $z_{0}(y)=h_{0}(y)$.

Put

$$
S=\left(S^{0} \times\{0\}\right) \cup S_{a}, \quad \text { with } S_{a} \text { given by }(3.9) .
$$

We have obviously: $S \subset M \cup M^{*}, \quad S \cap M^{*}=S \cap M^{\prime}=S_{a}$ and $\left(S \cap M^{*}\right) \cup M_{c}=S_{b}(\sec (3.10))$.

Hence, from Lemma 3 , we obtain the following conditions:

there is a retraction $\left(S \cap M M^{*}\right) \cup M_{e} \rightarrow S \cap M I^{*}$

and

$$
\text { there are no retractions } S \rightarrow S \cap M^{*} \text {. }
$$

Then, for the GSD-system $\left(X, R_{*}, \pi\right)$ with $X$ and $\pi$ given by (3.6) and (3.8) respectively, and for $M$ and $S$ given by (3.11) and (3.12), all assumptions of Theorem $R$, from Section 8 of Part $I$ of the present paper are satisfied. Hence, the conclusion of this theorem holds true, and then there is $\langle z, 0\rangle \in S \cap M$, such that $\pi(\langle z, 0\rangle)$ is contained in $M$; this means, however, that there is a $\varphi \in \mathscr{D}^{0}$ such that $\varphi(x, y) \epsilon(g(x, y), h(x, y))$ for every $\langle x, y\rangle$ belonging to $Q^{0}$.

3.3. Theorem 3. Let $Q^{0}$ be given by (2.1) and let $S^{0}$ be the same as in Section 3.1. Suppose, moreover, that $\mathscr{D}_{1}^{0}$ is a family of real functions, continuous and defined in $Q^{0}$, fulfilling conditions (i), (ii), (iii), (v) (see Section 3.1) with $\mathscr{D}^{0}$ replaced by $\mathscr{D}_{1}^{0}$, and, moreover, 
(iv') (a) there exists a positive constant $L_{1}$, such that $|\varphi(x, y)-\varphi(\tilde{x}, y)| \leqslant L_{1}|x-\tilde{x}|$ for $\varphi \in \mathscr{D}_{1}^{0}:\langle x, y\rangle,\langle\tilde{x}, y\rangle \epsilon Q^{0}$,

( $\beta$ ) for every $x \in[0, A)$, there is $L_{2}^{\prime}=L_{2}^{\prime}(x)$ such that $\sup \left\{L_{2}^{\prime}(t): 0 \leqslant t \leqslant x\right\}<\infty$ and $|\varphi(x, y)-\varphi(x, \tilde{y})|$ $\leqslant L_{2}^{\prime}(x)|y-\tilde{y}|$ for every $\varphi \in \mathscr{D}_{1}^{0}$ and $\langle x, y\rangle,\langle x, \tilde{y}\rangle \epsilon Q^{0}$.

(vi) There exists a continuous mapping $\lambda: Q^{0} \rightarrow R$, such that $|\varphi(x, y)| \leqslant \lambda(x, y)$ for every $\varphi \in \mathscr{D}_{1}^{0}$ and $\langle x, y\rangle \epsilon Q^{0}$.

Under these assumptions, there exists a function $\varphi \in \mathscr{D}_{1}^{0}$ such that $g(x, y)$ $<\varphi(x, y)<h(x, y)$ for $\langle x, y\rangle \epsilon Q^{0}$.

Proof. Let $\eta \in(0, A)$ be fixed. Consider

$$
Q_{\eta} \stackrel{\text { df }}{=} Q^{0} \cap([0, A-\eta) \times R) .
$$

It is easy to see that putting:

$$
\tilde{Q}^{0} \stackrel{\text { df }}{=} Q_{\eta} \quad \text { and } \quad \tilde{\mathscr{D}}^{0} \stackrel{\text { dt }}{=}\left\{\left.\varphi\right|_{\bar{Q}}: \varphi \in \mathscr{D}_{1}^{0}\right\},
$$

we obtain immediately the situation considered previously; all assumptions of Theorem 2 for $Q^{0}$ replaced by $\tilde{Q}^{0}$ and $\mathscr{D}^{0}$ replaced by $\tilde{D}^{0}$, are obviously satisfied. Hence, there is a function $\varphi \epsilon \tilde{\mathscr{D}}^{0}$, such that

$$
g(x, y)<\varphi(x, y)<h(x, y) \quad \text { for }\langle x, y\rangle \epsilon \tilde{Q}^{0} .
$$

Of course, in virtue of the continuity, we have

$$
g(x, y) \leqslant \varphi(x, y) \leqslant h(x, y)
$$

for $\langle x, y\rangle \epsilon \bar{Q}_{\eta}$.

Let us consider a strictly decreasing sequence of numbers $\eta_{n}$ belonging to the open interval $(0, A)$ and converging to zero. Put $Q_{n}=\bar{Q}_{\eta_{n}}$ $(n=1,2, \ldots)$, where $Q_{\eta_{n}}$ is defined by $(3.15)$ with $\eta$ replaced by $\eta_{n}$.

From the above considerations, we have the following obvious

Proposition. For every $n$ there is a $\varphi=\varphi_{n} \epsilon \mathscr{D}_{1}^{0}$ such that

$$
g(x, y) \leqslant \varphi(x, y) \leqslant h(x, y) \quad \text { for }\langle x, y\rangle \in Q_{n} .
$$

Then, for every $n$, the set

$$
E_{n}=\left\{\varphi \in \mathscr{D}_{1}^{0} ; g(x, y) \leqslant \varphi(x, y) \leqslant h(x, y) \text { for }\langle x, y\rangle \epsilon Q_{n}\right\}
$$

is non-empty. The sequence $\left\{E_{n}\right\}$ is decreasing (in the sense of inclusion).

Consider the space $\mathscr{F}$ of all real functions defined in $Q^{0}$, provided with the topology of almost uniform convergence.

Lemma 4. The set $\mathscr{D}_{1}^{0}$ is relatively compact in $\mathscr{F}$ (that is the closure $\overline{\mathscr{D}}_{\mathbf{1}}^{0}$ of the set $\mathscr{D}_{1}^{0}$ is compact). 
Proof. $1^{0}$ The closure of $\mathscr{D}_{1}^{0}$ is a set of continuous real functions, satisfying in every compact subset of $Q^{0}$, the Lipschitz condition with the same constants (depending only on the compact set); the functions are equibounded. $2^{\circ}$ For every $\langle x, y\rangle \epsilon Q^{0}$, the set $\left\{\varphi(x, y): \varphi \epsilon \overline{\mathscr{D}}_{1}^{0}\right\}$ is compact in $R$. Then, we can apply well-known theorems (see for instance Theorem 10.1 or 10.3 from the book [27]) and we obtain the compactness of $\overline{\mathscr{D}}_{1}^{0}$.

LEMAra 5. For every $n$, the set $E_{n}$ is closed in $\overline{\mathscr{D}}_{1}^{0}$ (which is - see Lemma 4 - compact space), and then $E_{n}$ is compact.

We omit the trivial proof of this lemma.

Return now to the proof of our theorem.

Using Lenmas 4 and 5 and the classical Cantor's theorem, we obtain the following conclusion:

$$
\bigcap_{n=1}^{\infty} E_{n} \neq \emptyset \text {. }
$$

Then, there is a function $\varphi \in \mathscr{D}_{1}^{0}$, such that $g(x, y) \leqslant \varphi(x, y) \leqslant h(x, y)$ for $\langle x, y\rangle \in Q_{n}$ for every $m$. Hence, there is a function $\varphi \in \mathscr{D}_{1}^{0}$, such that $g(x, y) \leqslant \varphi(x, y) \leqslant h(x, y)$ for $\langle x, y\rangle \in Q^{0}$. Observe finally, that for such a function $\varphi$, the equality $\varphi(x, y)=g(x, y)$ (or $\varphi(x, y)=h(x, y)$ ) for some $\langle x, y\rangle \in Q^{0}$ is impossible, in virtue of condition ( $\nabla$ ) (in this case we would have $\varphi E_{n}$ for some $n$ ). Hence, we have the strong inequalities and then there is a $\varphi \in \mathscr{B}_{1}^{0}$ such that $g(x, y)<\varphi(x, y)<h(x, y)$ for $\langle x, y\rangle \in Q^{0}$.

\section{APPLICATIONS OF THE RESULTS FROM CHAPTER 3} IN THE THEORY OF PARTIAL DIFFERENTIAL EQUATIONS OF THE FIRST ORDER

4.1. Notations and assumptions. Let us consider the following two sets :

$$
J=\left\{\langle x, y, z\rangle: 0 \leqslant x<\eta,|y| \leqslant b_{1}-\beta_{1} x,|z| \leqslant b_{2}-\beta_{2} \cdot x\right\}
$$

a subset of $R^{3}$, and

$$
Q^{*}=Q\left(\eta ; b_{1}, \beta_{1}\right) \quad(\text { see Section } 2.3),
$$

where $b_{1}, b_{2}, \eta \in R_{+}, \beta_{1}, \beta_{2} \in R_{*}$ are fixed and such that $\beta_{i} \eta<b_{i}(i=1,2)$.

Let $f$ be a real and continuous function defined in some set $U \subset R^{4}$ containing $J \times\left[-K^{1}, K^{1}\right]$, where $K^{1}$ is a positive constant.

We suppose, that $f$ fulfils condition (H) which is the conjunction of the two following conditions:

and

$$
\left(\mathrm{H}_{1}\right):(s) \cdot f\left(x, y,(s)\left(b_{2}-\beta_{2} x\right), 0\right)>-\beta_{2} \quad \text { for }\langle x, y\rangle \in Q^{*}
$$

$$
\begin{aligned}
\left(\mathrm{H}_{2}\right):(s) s_{q} f\left(x,(s)\left(b_{1}-\beta_{1} x\right),(s) s_{q}\left(b_{2}-\beta_{2} x\right), q\right)> & -\beta_{2}+\beta_{1}|q| \\
& \text { for } 0 \leqslant x<\eta,
\end{aligned}
$$


where $(s)=+,-, s_{q}=\operatorname{sign} q$.

Remark. The following condition is sufficient for $(\mathbf{H})$ :

$\left(\mathbf{H}^{*}\right):(s) \cdot s_{q} \cdot f\left(x, y(s) s_{q}\left(b_{2}-\beta_{2} x\right), q\right)>-\beta_{2}+\beta_{1}|q|, \quad\langle x, y\rangle \epsilon Q^{*}$.

In particular, if

$\left(\mathrm{H}^{* *}\right): f\left(x, y, b_{2}-\beta_{2} x, q\right)>-\beta_{2}+\beta_{1}|q| \quad$ for $\langle x, y\rangle \in Q^{*}, \quad$ and

$f(x, y, z, q)=-f(x, y,-z, q), \quad f(x, y, z, q)=-f(x, y, z,-q)$

for every $\langle x, y, z, q\rangle \in U$,

then condition $\left(\mathbf{H}^{*}\right)$ (and then $(\mathbf{H})$ also) is satisfied.

We assume furthermore, that $f$ fulfils the Lipschitz condition:

$$
|f(x, y, z, q)-f(x, y, \bar{z}, \bar{q})| \leqslant L_{1}^{*}|z-\bar{z}|+L_{2}^{*}|q-\bar{q}|
$$

for $\langle x, y, z, q\rangle,\langle x, y, \bar{z}, \bar{q}\rangle \epsilon J \times\left[-K^{1}, K^{1}\right]$.

Let $\hat{K}$ be a positive constant and let $\hat{G}$ be a subset the space of all real $C^{\mathbf{l}}$-functions defined in $\left[-b_{1}, b_{1}\right]$, having absolute values of derjvatives bounded by $\hat{K}$. TVe assume that $\{\varphi(y): \varphi \epsilon \hat{G}\}=\left[-\beta_{2}, \beta_{2}\right]$ for every $y \in\left[-b_{1}, b_{1}\right]$, and that $G$ is connected as a subset of $\mathscr{C}\left(\left[-b_{1}, b_{1}\right], R\right)$ (see Section 2.1).

4.2. Applications of Theorem 2 to a Cauchy problem given by the function $f$. Consider the following Cauchy problem:

$$
\begin{gathered}
z_{x}=f\left(x, y, z, z_{y}\right), \\
z(0, y)=\varphi(y),
\end{gathered}
$$

where $\varphi$ is a real $C^{1}$-function defined in $\left[-b_{1}, b_{1}\right]$.

THEOREM 4. Assume all conditions introduced above in the previous Section 4.1. Assume, moreover, that for each $\varphi \epsilon \hat{G}$ there is a solution $z=z[\varphi]$ of (4.3)-(4.4), which is a $C^{1}$-function defined in $Q^{*}$, such that $\left|z_{y}(x, y)\right| \leqslant K^{1}$ for $\langle x, y\rangle \in Q^{*}$. Assume finally that

$$
K^{1}<\left(b_{2}-\beta_{2} \eta\right) / b_{1} .
$$

Under the above assumptions, there is a function $\varphi^{0} \epsilon \hat{G}$, such that $z^{0}$ $=z\left[\varphi^{0}\right]$ fulfils the inequality

$$
\left|z^{0}(x, y)\right|<b_{2}-\beta_{2} x \quad \text { for }\langle x, y\rangle \in Q^{*} .
$$

Proof. First of all observe that the mapping $\varphi \mapsto z[\varphi]$ is really well defined, since in virtue of the assumptions, we have for every $\varphi \in \dot{G}$ the existence (explicitly assumed) of a solution $z=z[\varphi]$; but this solution is therefore unique, since the function $f$ satifies the Lipschitz condition (for details, see for instance Szarski [32]). 
Putting $g(x, y)=-b_{2}+\beta_{2} x, h(x, y)=+b_{2}-\beta_{2} x, A=\eta, Q^{0}=Q^{*}$, $B=b_{2}, S^{0}=\hat{G}, \mathscr{D}^{0}=\left\{z[\varphi]: \varphi \in \mathbb{S}^{0}\right\}$, we can easily verify that all assumptions of Theorem 2 are satisfied for these $Q^{0}, S^{0}, \mathscr{D}^{0}$; in particular condition $(\nabla)$ is a consequence of $(\mathrm{H})$ and (4.5). Hence the conclusion of Theorem 2 holds true; this means that the conclusion of our Theorem 4 holds true.

4.3. A modification of Theorem 3. Let us consider a subset $\mathscr{D}_{2}^{0}$ of $\mathscr{C}\left(Q^{0}, R\right)$, with $Q^{0}=Q^{*}$ given by (4.2). Suppose that $\mathscr{D}_{2}^{0}$ fulfils the condjtions assumed in 3.1 and 3.4 for $\mathscr{D}_{1}^{0}$ with only one change: the set $S^{0}$ is replaced by $S_{2}^{0}$ which is a set of real functions defined on $[-B, B]$, fulfilling the Lipschitz condition with the constant $K$, and such that for every $y \in[-B, B]$, the set $\left\{\lambda(y): \lambda \in \mathbb{S}_{2}^{0}\right\}$ is a closed interval contained in the interval $[g(0, y), h(0, y)](g, h$ given previously (see Section 3.1)), and, moreover, $S_{2}^{0}$ is connected in $\mathscr{C}([-B, B], R)$ (for the last condition it is sufficient to assume that: $\left(u, v \in S_{2}^{0}, u \neq v\right) \Rightarrow(u(y) \neq v(y)$ for $\left.|y| \leqslant B)\right)$.

Define functions $g^{*}$ and $h^{*}$ as follows:

$$
\begin{gathered}
g^{*}(x, y)=\max \left(\inf \left\{\varphi(x, y): \varphi \in S_{2}^{0}\right\}, g(x, y)\right), \quad\langle x, y\rangle \in Q^{*}, \\
h^{*}(x, y)=\min \left(\sup \left\{\varphi(x, y): \varphi \in \mathbb{S}_{2}^{0}\right\}, h(x, y)\right),\langle x, y\rangle \in Q^{*}
\end{gathered}
$$

THEOREM 5. Suppose all assumptions of Theorem 3 for $S_{2}^{0}$ and $\mathscr{D}_{2}^{0}$ substituted in the place of $S^{0}$ and $\mathscr{D}_{1}^{0}$ respectively. Assume, moreover, that either

$$
g^{*}(x, y)>g(x, y) \quad \text { and } \quad h(x, y)>h^{*}(x, y) \quad \text { for }\langle x, y\rangle \in Q^{*},
$$

or

(II) there exist functions $\varphi, \psi \in S_{2}^{0}$ and there are $\langle x, y\rangle,\langle u, v\rangle$ belonging to $Q^{*}$, such that $\tilde{\varphi}(x, y)=g^{*}(x, y), \tilde{\psi}(u, v)=h^{*}(u, v)$

for $\tilde{\varphi}, \tilde{\psi} \epsilon \mathscr{D}_{2}^{0}$, such that $\tilde{\varphi}(0, y)=\varphi(y), \tilde{\psi}(0, y)=\psi(y)$ for $y \in[-B, B]$.

Under the above assumptions, there is a function $\varphi \in \mathscr{D}_{2}^{0}$ such that $g(x, y)$ $\left\langle\varphi(x, y)<h(x, y)\right.$ for $\langle x, y\rangle \epsilon Q^{*}$.

In order to prove this theorem, we apply the same reasoning as in the proof of Theorem 3, with the following simple modifieations: we consider the sets

$$
\left(S_{a}\right)_{2}=\left\{\left\langle\inf \left\{\varphi(0, y): \varphi \in \mathscr{D}_{2}^{0}\right\}, 0\right\rangle,\left\langle\sup \left\{\varphi(0, y): \varphi \in \mathscr{D}_{2}^{0}\right\}, 0\right\rangle\right\}
$$

and

$$
\left(S_{b}\right)_{2}=\left(S_{2}^{0} \times\{0\}\right) \cup\left(S_{a}\right)_{2} \quad \text { in the place of the sets } S_{a} \text { and } S_{b},
$$

and corresponding mappings which are obvious modifications of the mappings introduced in Iemma 3 . We omit the elenıentary details. 
Remark. Formally, Theorem 5 is a generalization of Theorem 3 (for $Q^{0}=Q^{*}$ of course; a general theorem for arbitrary $Q^{0}$ is also true), Theorem 3 was, however, proved with all details, since the general idea of the proof (being the same in both cases) can be presented in a simpler manner in a little simpler case.

We will give below an application of Theorem 5 .

4.4. An example. We use here the notation from Section 4.1 and 4.2 . In particular $K^{1}$ is the positive constant introduced above. Suppose that the constants $D, K^{0}, D^{1}$ are positive and such that $K^{0}<K^{1}, D^{1}<D$.

Let us assume, moreover, that

$$
\eta=\left(K^{0}\right)^{2} /\left(32\left(D+K^{0}+1\right)\right) .
$$

Suppose that $f$ is a real function defined for $\langle x, y, z, q\rangle$ belonging to $\left[-K^{1}, K^{1}\right]^{4} \subset R^{4}$, continuous together with its derivatives $f_{y}, f_{z}, f_{q}$. The derivatives $f_{y}, f_{z}, f_{q}$ fulfil the Lipschitz condition with respect to $\langle y, z, q\rangle$ with the constant $D^{1}$. Furthermore $|f|,\left|f_{y}\right|,\left|f_{z}\right|,\left|f_{\alpha}\right|<D^{1}$ in the set $\left[-K^{1}, K^{1}\right]^{4}$. Let $\hat{G}_{1}$ be a subset of the set of all real $C^{1}$-functions $\varphi$, defined in $\left[-K^{1}, K^{1}\right]$, such that $|\varphi(0)|<K^{0} / 4,\left|\varphi^{\prime}(0)\right|<K^{0} / 4,\left|\varphi^{\prime}(y)\right|$ $<D^{1}$ for $|y| \leqslant K^{1}$ and $\left|\varphi^{\prime}(y)-\varphi^{\prime}(\tilde{y})\right| \leqslant D^{1}|y-\tilde{y}|$ for $|y|,|\tilde{y}| \leqslant K^{1}$. TVe assume that $\hat{G}_{1}$ is connected as a subset of $\mathscr{C}\left(\left[-b_{1}, b_{1}\right], R\right)$, and that for every $y \in\left[-K^{1}, K^{1}\right]$, the set $\left\{\varphi(y): \varphi \epsilon \hat{G}_{1}\right\}$ is a closed interval. In the sequel we will use the following existence theorem:

Tuieorem E (cf. Ważewski [39], Szarski [32]). Under the above assumptions, for every $\varphi \epsilon \hat{G}_{1}$, there exists exactly one solution $z=z[\varphi]$ of (4.3)-(4.4), defined and of the class $C^{1}$ in the set:

$$
\tilde{Q}=\left\{\langle x, y\rangle: 0 \leqslant x<\eta,|y| \leqslant b_{1}-D x\right\},
$$

where

$$
b_{1}=K^{0} /(4 D+4) \text {. }
$$

Lenras 6. Assume that

$$
\begin{gathered}
K^{0}<K^{1}<4(D+1), \\
\beta_{2} K<8(D+1)^{4}\left(4 D+4-K^{1}\right) .
\end{gathered}
$$

Then, every solution $z=z[\varphi]$ of $(4.3)-(4.4)$, with $\varphi \epsilon \hat{G}_{1}$, fulfils the following condition: if $z(x, y)=K^{1}-\beta_{2} x$ (resp. $z(x, y)=-K^{1}+\beta_{2} x$ ) for some $\langle x, y\rangle \epsilon \tilde{Q}$, then $z(x, \bar{y}) \neq-K^{1}+\beta_{2} x \quad$ (resp. $\left.z(x, \bar{y}) \neq K^{1}-\beta_{2} x\right)$ for every $\bar{y}$ such that $|\bar{y}| \leqslant b_{1}-D x$, with $b_{1}$ given by (4.7).

Proof. Conditions (4.8) and (4.9) imply the inequality

$$
K^{1}<4(D+1)-\beta_{2} K^{0}\left(8(D+1)^{4}\right)^{-1} \quad \text { and then } K^{1}<\left(K^{1}-\beta_{2} \eta\right) / b_{1} .
$$


Hence, for every $\varphi \in \hat{G}_{1}$, the unique solution $z=z[\varphi]$ of $(4.3)-(4.4)$ fulfils the inequality $\left|z_{y}(x, y)\right|<\left(K^{1}-\beta_{2} \eta\right) / b_{1}$, which gives inmediately the conclusion of the lemma.

Remark. In Theorem 6 (see below) we will assume - among the other conditions - condition (H) for $f$ (see Section 4.1). A necessary condition for these assumptions is the following inequality:

$$
D K^{1}<D^{1} \text {. }
$$

If (4.8) and (4.10) are satisfied, then $K^{1}$ must be such that

$$
K^{1}<2\left(1+\left(1+\left(D^{1}\right)^{2}\right)^{1 / 2}\right) .
$$

Thus, the natural conditions in the sequel would be (4.10) and (4.11); we will assume them.

THEonem 6. If conditions (4.8)-(4.11) are satisfied for the constants introduced above, the function $f$ satisfies $(\mathrm{H})$ and

( $\left.{ }^{0}\right)$ there exist $\varphi, \psi \in \hat{\theta}_{1}$, such that

$$
\begin{aligned}
z[\varphi](x, y)=K^{1}-\beta_{2} x, z[\psi](u, v)= & -K^{1}+\beta_{2} u \\
& \text { for some }\langle x, y\rangle,\langle u, v\rangle \in \tilde{Q},
\end{aligned}
$$

then there exists a function $\lambda \in \hat{G_{1}}$, such that

$$
|z[\lambda](x, y)|<K^{1}-\beta_{2} x \quad \text { for }\langle x, y\rangle \epsilon \tilde{Q} .
$$

In order to prove this theorem we apply Theorem $\tilde{5}$; here $g(x, y)$ $=-K^{1}+\beta_{2} x, h(x, y)=+K^{1}-\beta_{2} x$. Condition $\left({ }^{0}\right)$ implies directly assumption (II) of Theorem $\tilde{\mathbf{5}}$.

COROLLARY. Under the assumptions of Theorem 6 without $\left({ }^{\circ}\right)$, we have the following conclusion: among the following three conditions:

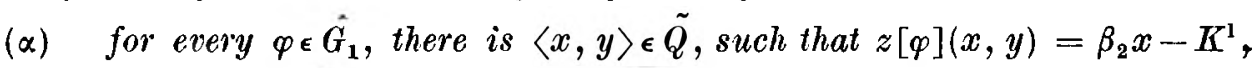

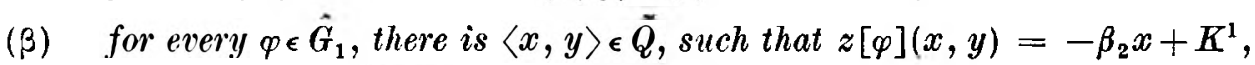

$(\gamma)$ there exists $\hat{\varphi} \in G_{1}$, such that $|z[\varphi](x, y)|<K^{1}-\beta_{2} x$ in $\tilde{Q}$, at least one holds true.

\section{EXTENSIONS OF THE RESULTS FROM CHAPTER 4 FOR SYSTEMS OF DIFFERENTIAL EQAUTIONS}

One can extend the previous results from Chapter 4 to systems of differential equations. We will give below an example of such extensions.

Let $k, b^{i}$ and $\delta$ be positive constants, $\beta^{i}$ be non-negative numbers $(i=0,1,2)$ such that $b^{i}-\beta^{i} \delta>0 \quad(i=0,1,2)$. Put $Q^{\prime}=\{\langle x, y\rangle: 0$ $\left.\leqslant x<\delta,|y| \leqslant b^{0}-\beta^{0} x\right\}$. Let $f^{i}: Q^{\prime} \times R^{4} \rightarrow R$ be continuous $(i=1,2)$. 
Suppose that $f^{i}=f^{i}\left(x, y, z_{1}, z_{2}, q_{1}, q_{2}\right)$ are such that $(s) f^{1}\left(x, y,(s)\left(b^{1}-\beta^{1} x\right), z_{2}, 0, q_{2}\right)>-\beta^{1} \quad$ for $\langle x, y\rangle \in Q^{\prime}, q_{2} \in R$, $\left|z_{2}\right| \leqslant b^{2}-\beta^{2} x$

$(s) s^{1} f^{1}\left(x,(s)\left(b^{0}-\beta^{0} \cdot x\right),(s) s^{1}\left(b^{1}-\beta^{1} x\right), z_{2}, q_{1}, q_{2}\right)>-\beta^{1}+\beta^{0}\left|q_{1}\right|$ for $0 \leqslant x<\delta,\left|z_{2}\right| \leqslant b^{2}-\beta^{2} x,\left|q_{1}\right| \leqslant k, q_{2} \in R$;

$(s) f^{2}\left(x, y, z_{1},(s)\left(b^{2}-\beta^{2} x\right), q^{1}, 0\right)<-\beta^{2} \quad$ for $\langle x, y\rangle \in Q^{\prime},\left|q_{1}\right| \leqslant k$, $\left|z_{1}\right| \leqslant b^{1}-\beta^{1} x ;$

$$
\begin{array}{r}
(s) s^{2} f^{2}\left(x,(s)\left(b^{0}-\beta^{0} x\right), z_{1},(s) s^{2}\left(b^{2}-\beta^{2} x\right), q_{1}, q_{2}\right)<-\beta^{2}+\beta^{0} \mid q_{2} ! \\
\text { for } 0 \leqslant x<\delta,\left|z_{1}\right| \leqslant b^{1}-\beta^{1} x,\left|q_{1}\right| \leqslant k, q_{2} \in R ;
\end{array}
$$

where $(s)=+,-$ and $s^{i}=\operatorname{sign}\left(q^{i}\right)(i=1,2)$.

Let $S_{0}^{\prime}$ be a set of $C^{1}$-functions defined on $\left[-b^{0}, b^{0}\right]$ such that: $\left(\lambda, \varrho \in S_{0}^{\prime}\right.$, $\lambda(y)=\varrho(y)$ for some $\left.y,|y| \leqslant b^{0}\right) \Rightarrow(\lambda=\varrho)$, and $\left\{\lambda(y): \lambda \in \mathbb{S}_{0}^{\prime}\right\} \supset\left[-b^{0}, b^{0}\right]$ for every $y \in\left[-b^{0}, b^{0}\right]$ and $\left\{\lambda(y): \lambda \in S_{0}^{\prime}\right\}$ is a closed interval for every $y \epsilon\left[-b^{0}, b^{0}\right]$. Suppose that for every pair $\lambda=\left\langle\lambda_{1}, \lambda_{2}\right\rangle \epsilon S^{\prime}=S_{0}^{\prime} \times S_{0}^{\prime}$, there is exactly one solution $z[\lambda]=\left\langle z^{1}[\lambda], z^{2}[\lambda]\right\rangle$ of problem $(\mathrm{P})$ :

$z_{x}^{i}=f^{i}\left(x, y, z^{1}, z^{2}, z_{y}^{1}, z_{y}^{2}\right)(i=1,2)\langle x, y\rangle \in Q^{\prime}$ with the initial conditions $z^{i}(0, y)=\lambda_{i}(y)$ for $|y| \leqslant b^{0}(i=1,2)$,

defined and continuous together with the first deriratives in $Q^{\prime}$; suppose that the mapping $z \mapsto z[\lambda]$ is continuous.

Assume finally that if $\lambda_{1} \in S_{0}^{\prime}$ and $\left|\lambda_{1}(y)\right| \leqslant b^{1}$ for $|y| \leqslant b^{0}$, then for every $\lambda_{2} \in S_{0}^{\prime}$, the solution $z[\lambda]$ of problem $(P)$ fulfils the inequality

$$
\left|z_{y}^{1}(x, y)\right| \leqslant k .
$$

THEOREM 7. Assume that all conditions introduced above are satisfied. Suppose, moreover, that $k<\left(b^{1}-\beta^{1} \delta\right) / b^{0}$.

Then there exists a $\lambda=\left\langle\lambda_{1}, \lambda_{2}\right\rangle \in S^{\prime}$ such that the (unique) solution $z[\lambda]$ of problem (P), fulfils the inequalities $\left|z^{i}(x, y)\right|<b^{i}-\beta^{i} x$ for $\langle x, y\rangle \in Q^{\prime}, i=1,2$.

This theorem is a corollary of the two following lemmas:

Lemma 7. For every $\lambda_{2} \epsilon S_{0}^{\prime}$ such that $\left|\lambda_{2}(y)\right|<b^{2}$ for $|y| \leqslant b^{0}$, there exists $\lambda_{1} \in S_{0}^{\prime}$ such that the pair $\left\langle\lambda_{1}, \lambda_{2}\right\rangle=\lambda$ gives the solution $z=z[\lambda]$ of $(\mathrm{P})$, which fulfils the inequality

$$
\left|z^{1}(x, y)\right|<b^{1}-\beta^{1} x \quad \text { for }\langle x, y\rangle \in Q^{1} .
$$

LeMma 8. If $\lambda=\left\langle\lambda_{1}, \lambda_{2}\right\rangle \in S^{\prime}$ is such that $\left|\lambda_{2}(y)\right|<b^{2}$ for $|y| \leqslant b^{0}$ and $z=z[\lambda]$ fulfills the above inequality $(*)$, then

$$
\left|z^{2}(x, y)\right|<b^{2}-\beta^{2} x \quad \text { for }\langle x, y\rangle \in Q^{\prime} .
$$

We will limit ourselves to giving only an outline of the proof of Lemma 7. The proof of Lemma 8, as well as the rest of the proof of Theorem 7, is very simple or even trivial. 
In order to prove Lemma 7 , observe that for every $\lambda \in S^{\prime}$ such that $\lambda_{i}(y)<b^{i}$ for $|y| \leqslant b^{0}(i=1,2)$, and for

$x^{0} \stackrel{d f}{=} \sup \left\{x \in[0, \delta]:\left|z^{i}[\lambda](x, y)\right|<b^{i}-\beta^{i} \cdot x\right.$ for $\left.\langle x, y\rangle \in Q^{\prime}, i=1,2\right\}$

we have the following implication:

if $x^{0}<\delta$, then the following three conditions are satisfied:

(a) $\left|z^{2}[\lambda]\left(x^{0}, y\right)\right|<b^{2}-\beta^{2} x^{0}$ for $|y| \leqslant b^{0}-\beta^{0} x^{0}$,

(b) $\left|z^{1}[\lambda]\left(x^{0}, y\right)\right|=b^{1}-\beta^{1} x^{0}$ for some $y$ such that $|y| \leqslant b^{0}-\beta^{0} x^{0}$,

(c) there is a positive number $\eta$ such that for each

$x \in\left(x^{0}, x^{0}+\eta\right)$, there exists $\tilde{y}$ such that $|\tilde{y}| \leqslant b^{0}-\beta^{0} x$ and

$\left|z^{1}[\lambda](x, \tilde{y})\right|>b^{1}-\beta^{1} x \quad$ (more precisely: $z^{1}[\lambda](x, \tilde{y})>b^{1}-\beta^{1} \cdot x$ if $z^{1}[\lambda]\left(x^{0}, y\right)=b^{1}-\beta^{1} x^{0}$ for some $y$ (see $\left.(\mathrm{b})\right)$, and $z^{1}[\lambda](x, \tilde{y})$ $<-b^{1}+\beta^{1} x$ if $z^{1}[\lambda]\left(x^{0}, y\right)=-b^{1}+\beta^{1} x^{0}$ for some $\left.y\right)$.

Using Theorem 3 we finish the proof of Lemma 7 .

\section{FURTHER APPLICATIONS OF THE MAIN RESULTS FROM THE FIRST PART IN THE THEORY OF PARTIAL DIFFERENTIAL EQUATIONS}

6.1. Notations, preliminary assumptions and propositions. A special case of the existence theorem of $T$. Waziewski

6.1 (i) Let constants $K, K_{1}, M, M_{1}$ be given in such a way that $0<K<K_{1} \leqslant M_{1}<M$. Put

$$
\begin{gathered}
\delta=\frac{K^{2}}{32 \cdot(M+K+1)^{5}}, \\
\mu=\frac{K}{4 \cdot(M+1)}, \\
\alpha=2+M_{1}+K
\end{gathered}
$$

and, for $x \in[0, \delta]$ :

$$
N(x)=M_{1} \cdot \frac{1+\alpha\left(\alpha+M_{1}\right) \cdot x}{1-M_{1}\left(\alpha+M_{1}\right) \cdot x .} .
$$

It is obvious that $N(x)>0$ for every $x \in[0, \delta]$ and that $N(0)=M_{1}$.

Moreover, $N$ is an increasing function.

Denote by $\mathscr{P}$ the set of all real $C^{1}$-functions $\varphi$ defined in $[-\mu, \mu]$, such that $\left|\varphi^{\prime}(y)\right| \leqslant M_{1}$ and $\left|\varphi^{\prime}(y)-\varphi^{\prime}(\tilde{y})\right| \leqslant M_{1}|y-\tilde{y}|$ for $|y|,|\tilde{y}| \leqslant \mu$.

Finally, we put

$$
\text { - } \hat{Q}=\{\langle x, y\rangle: 0 \leqslant x \leqslant \delta,|y| \leqslant \mu-M x\} .
$$

6.1 (ii) From the known results of $T$. Ważewski (cited partially in Section 4.4 as Theorem E) given in papers [39] and [40] (see also Szarski [32]), there follows 
THEOREM W. Let the constants $K, K_{1}, M, M M_{1}, \delta, \mu$ be as above and let a function $f:\left[0, K_{1}\right] \times R^{3} \rightarrow\left[-M_{1}, M_{1}\right]$ be continuous. Suppose also that $f=f(x, y, z, q)$ has the first partial derivatives $f_{y}, f_{z}, f_{q}$ which are continuous functions of $\langle x, y, z, q\rangle$, such that $\left|f_{y}(x, y, z, q)\right|,\left|f_{z}(x, y, z, q)\right|$, $\left|f_{q}(x, y, z, q)\right| \leqslant M_{1}$ for each $\langle x, y, z, q\rangle$ belonging to their domain of de. finition. Assume, moreover, that $f_{y}, f_{z}, f_{q}$ satisfy the Lipschitz condition with respect to $\langle y, z, q\rangle$ with the constant $M_{1}\left(\left|f_{y}(x, y, z, q)-f_{y}(x, \tilde{y}, \tilde{z}, \tilde{q})\right|\right.$ $\leqslant M_{1}(|y-\tilde{y}|+|z-\tilde{z}|+|q-\tilde{q}|)$ etc. $)$.

Then, for each $\phi \in \mathscr{P}$, there exists exactly one real, $C^{1}$-function $z=z[\varphi]$ defined in $\hat{Q}$, which is the (unique) solution of the problem:

$$
\begin{aligned}
\partial z / \partial x & =f(x, y, z, \partial z / \partial y), \\
z(0, y) & =\varphi(y),
\end{aligned}
$$

and fulfilling, moreover, the following conditions:

$$
\begin{array}{cl}
\left|z_{y}(x, y)\right| \leqslant K_{1} & \text { for }\langle x, y\rangle \in \hat{Q}, \\
\left|z_{y}(x, y)-z_{y}(x, \tilde{y})\right| \leqslant N(x)|y-\tilde{y}| & \text { for }\langle x, y\rangle \in \hat{Q} .
\end{array}
$$

The existence and the uniqueness of solutions $z=z[\varphi]$ of $(6.6)-(6.7$; for $\varphi \in \mathscr{P}$ follows directly from T. Ważewski's general result from [35. (see also [28]), stated for systems of differential equations. Condition (6.9) is not given explicitly in [39] (it is stated only that $z_{y}$ is lipschitzian with respect to $y$ ), but it can be immediately obtained from the estimates for second derivatives $z_{z y}$ of solutions of (6.6)-(6.7) (under stronger conditions on regularity of $f$ and $\varphi$ ), given in [40] (Remark 7, p. 177).

We will omit the details.

Technical arguments leading to estimate $(6.8)$ can be easily founc in [39] and [40].

6.1 (iii) Let now $c$ and $d$ be two constants, such that

$$
c \geqslant 0, \quad d>0, \quad \delta c<d \quad(\delta \text { given by (6.1)). }
$$

We put

$$
r(x)=\mu^{\prime}\left(\frac{d}{d-c x} \frac{M_{1}}{N(x)}\right)^{1 / 2} \quad \text { for } x \in[0, \delta]
$$

and

$$
s(x)=r(x) \cdot(d-c x) / d \quad \text { for } x \in[0, \delta] .
$$

In virtue of the assumptions on $d, c$ and $\delta$ and in virtue of the definitions of $\mu$ and $N(x)$, we have the following obvious properties of the functions $r$ and $s$ :

$$
\begin{aligned}
& s(x)>0 \quad \text { for } x \in[0, \delta], \quad r(x) \leqslant \mu, \quad s \text { is decreasing, } \\
& s(0)=\mu .
\end{aligned}
$$


Put

$$
Q(s) \stackrel{\text { df }}{=}\{\langle x, y\rangle: 0 \leqslant x<\delta,|y| \leqslant s(x)\} .
$$

In virtue of $(6.13)$, we have

$$
Q(s) \subset \hat{Q} .
$$

6.I (iv) Let $x \epsilon[0, \delta]$ be fixed. For a real $C^{1}$-function $u:[-s(x), s(x)]$ $\rightarrow[-d+c x, d-c x]$, we define a function $w=w^{u, x}:[-\mu, \mu] \rightarrow R$ by the following formula

$$
w^{u, x}(y)=\frac{d}{d-c x} \cdot u\left(\frac{s(x)}{\mu} y\right) \quad \text { for } y \in[-\mu, \mu] .
$$

We have the following simple

Proposition 1. If $u$ is a $C^{1}$-function from $[-s(x), s(x)]$ into $[-d+c x$, $d-c x]$ and $\left|u^{\prime}(y)\right| \leqslant K_{1},\left|u^{\prime}(y)-u^{\prime}(\tilde{y})\right| \leqslant N(x)|y-\tilde{y}|$, then $w=w^{u, x}$ is of the class $C^{1}$ and: (a) $|w(y)| \leqslant d$, (b) $\left|w^{\prime}(y)\right| \leqslant K_{1}$, (c) $\left|w^{\prime}(y)-w^{\prime}(\tilde{y})\right|$ $\leqslant M_{1}|y-\tilde{y}|$ for $|y|,|\tilde{y}| \leqslant \mu$.

Proof. Condition (a) is trivial. In order to prove (b), we calculate:

$$
\left|w^{\prime}(y)\right|=\left|\frac{d}{d-c x} \cdot \frac{s(x)}{\mu} \cdot u^{\prime}\left(\frac{s(x)}{\mu} y\right)\right| \leqslant\left|\frac{r(x)}{\mu}\right| K_{1} \leqslant K_{1} \quad \text { (see (6.13)). }
$$

Using (6.10) we can prove (c):

$$
\begin{array}{r}
\left|w^{\prime}(y)-w^{\prime}(\tilde{y})\right| \leqslant \frac{d}{d-c x} \cdot \frac{s(x)}{\mu} \cdot \frac{s(x)}{\mu} \cdot N(x)|y-\tilde{y}|=\frac{d-c x}{d} \cdot\left(\frac{r(x)}{\mu}\right)^{2} \times \\
\times N(x) \cdot|y-\tilde{y}|=M_{1}|y-\tilde{y}| .
\end{array}
$$

6.1 (v) Propositron 2. Let us consider the set $\mathscr{P}$ as a subset of $\mathscr{C}([-\mu, \mu], R)$ provided with its usual topology (see Section 2.1).

Then, the mapping $\mathscr{P} \in \varphi \mapsto z[\varphi] \epsilon \mathscr{C}(\hat{Q}, R)$ is continuous.

This is an immediate consequence of well-known theorems on continuous dependence of solutions on initial data.

Proposition 3. If we denote by $\mathscr{C}_{x}^{1}$ the set of all $C^{1}$-functions $u:[-s(x), s(x)] \rightarrow[-d+c x, d-c x]$, then we obtain the following conclusion: the mapping $\mathscr{C}_{x}^{1} \ni u \mapsto w^{u, x} \in \mathscr{C}([-\mu, \mu], R)$ is continuous.

The proof is trivial.

6.1 (vi) In the sequel we will need the following additional assumption $(A)$ :

If a solution $z=z[\varphi]$ of $(6.6)-(6.7)$ with some $\varphi \in \mathscr{P}$, is such that: $1^{0}|z(x, y)|<d-c x$ for $0 \leqslant x<x^{0}<\delta$ and $2^{\circ}\left|z\left(x^{0}, y^{0}\right)\right|=d-c x^{0}$ for some $y^{0} \in\left[-s\left(x^{0}\right), s\left(x^{0}\right)\right]$, then there exists $\eta>0$ such that for every $x \in\left(x^{0}, x^{0}+\eta\right)$, there is $y \in[-s(x), s(x)]$, for which $|z(x, y)|>d-c x$. 
This condition (A) is satisfied, for instance, if $f$ fulfils the following conditions :

$$
f(x, y, d-c x, q)>d-c x, \quad f(x, y-d+c x, q)<-d+c x,
$$

and

$$
\begin{aligned}
& f(x, s(x), d-c x, q)>-s^{\prime}(x) \cdot q+d-c x \text { for } q \geqslant 0, \\
& f(x,-s(x), d-c x, q)>s^{\prime}(x) \cdot q+d-c x \text { for } q \leqslant 0, \\
& f(x, s(x),-d+c x, q)<-s^{\prime}(x) \cdot q-d+c x \text { for } q \geqslant 0, \\
& f(x,-s(x),-d+c x, q)<s^{\prime}(x) \cdot q-d+c x \text { for } q \leqslant 0, \\
& \text { for every }\langle x, y\rangle \in Q(s)
\end{aligned}
$$

and, moreover,

$$
s^{\prime} \text { is the increasing function. }
$$

Notice, that (6.19) is - in particular - satisfied, if for instance $K$ and $c$ are sufficiently small. Indeed, it is easy to see that the second derivative $s^{\prime \prime}$ is positive if $c=0$ and $\delta$ are sufficiently small; this means that for every $K_{1}, M, M_{1}$, there are positive (sufficiently small) numbers $o$ and $K$, such that $s^{\prime}$ is increasing, or if $c=0$ there is a positive (sufficiently small) number $K$, such that $s^{\prime}$ is increasing. Of course, the above conditions, are not necessary for (A).

The author would like to note here that some numerical calculations necessary for checking that (6.19) is satisfied for a non-empty class of functions $s$ (that is for a non-empty class of constants $K, K_{1}, \ldots$, etc.) were done by Mr J. Marciński.

6.2. The main result. Theorem 8. Let us put

$$
\mathscr{P}^{0}=\{\varphi \in \mathscr{P}:|\varphi(y)| \leqslant d \text { for } y \in[-\mu, \mu]\} .
$$

Consider the set $\mathscr{D}(s)$ of all functions $z: Q(s) \rightarrow R$, of the form: $z$ is the restriction to the set $Q(s)$ of some solution $z=z[\varphi]$ of $(6.6)-(6.7)$ with some $\varphi \in \mathscr{P O}^{0}$; in the other words, we consider the set $\mathscr{D}(s)$ (a subset of $\mathscr{C}(Q(s), R)$ ) defined formally as follows:

$$
z \in \mathscr{D}(s) \stackrel{\text { df }}{\Leftrightarrow} \text { there exists } \varphi \in \mathscr{P}^{0} \text {, such that } z=\left.z[\varphi]\right|_{Q(s)} .
$$

In virtue of the equality $r(0)=s(0)=\mu$ (see (6.13)) and the uniqueness property, we can state the following

Proposition 4. For every $\varphi \in \mathscr{P}$, there exists exactly one function $z$, belonging to $\mathscr{D}(s)$ such that $z=\left.z[\varphi]\right|_{Q(s)}$, where $z[\varphi]$ denotes as reviously the unique solution of $(6.6)-(6.7)$. 
We will write

$$
\left.z\{\varphi\} \stackrel{\mathrm{df}}{=} z[\varphi]\right|_{Q(s)} \cdot
$$

In virtue of Propositions 2 and 4, we have the following obvious:

Proposition 5. The mapping $\mathscr{P} \ni \varphi \mapsto z\{\varphi\} \epsilon \mathscr{C}(Q(s), R)$ is well defined and is continuous.

Now, we can state our main result concerning the existence of a solution $z$ of $(6.6)-(6.7)$ having absolute value bounded by $d-c x$ in $Q(s)$.

THEOREM 8. Suppose that $f$ fulfils all conditions assumed in Theorem W, and, moreover, that condi ion (A) from Section 6.1 ( $\mathrm{vj}$ ) is also satisfied. Then there exists a function $\varphi$ belonging to $\mathscr{P}$ such that the function $z=z\{\varphi\}$ (see (6.22)) fulfils the inequality

$$
|z(x, y)| \leqslant d-c x \quad \text { for }\langle x, y\rangle \in Q(s) \text {. }
$$

6.3. Proof of Theorem 8. We will use the same method as in the proof of Theorem 2 (and Theorem 3).

Put:

$$
Q[s]=Q(s) \cup\{\langle x, y\rangle: 0 \leqslant x, y \leqslant s(\delta)\}
$$

and consider the set $\mathscr{D}[s]$ of all extensions of functions $z \in \mathscr{D}(s)$ on the set $Q[s]$. More precisely, if $z^{0} \in \mathscr{D}(s)$, then we put for $\langle x, y\rangle \in Q[s]$ :

$$
\begin{array}{ll}
z(x, y)=z^{0}(x, y) & \text { for }\langle x, y\rangle \in Q(s), \\
z(x, y)=\lim _{x \rightarrow \infty} z^{0}(x, y) & \text { for }\langle x, y\rangle \in Q[s] \backslash Q(s)
\end{array}
$$

and we denote by $\mathscr{D}[s]$ the set of all functions $z$, such that there is $z^{0} \in \mathscr{D}(s)$, for which $z$ is the extension given by (6.25).

As in Section 3.2 we denote by $Q[s]_{(x)}$ and $Q[s]_{x}$ the sets $Q[s] \cap(\{x\} \times R)$ and $Q[s] \cap([0, x] \times R)$ respectively.

By $z_{(x)}$ and $z_{x}$ we denote the restrictions of $z \in \mathscr{D}[s]$ to the $\operatorname{sets} Q[s]_{(x)}$ and $Q[s]_{x}$ respectively.

The context will always, we hope, make it impossible to confuse these with the first derivatives $\partial z / \partial x$.

Finally we define $Z(s)=\left\{\varphi_{x}: \varphi \in \mathscr{D}[s], x \in R_{*}\right\}$ and we consider a subset $X(s)$ of $Z(s) \times R_{*}$ defined by the following formula:

$X(s)=\left\{\langle u, x\rangle: x \in R_{*}\right.$ there exists $z \in \mathscr{D}[s]$ such that $\left.z_{x}=u\right\}$.

We introduce in $X(s)$ the topology in the same way as in Section 3.2, using the family of neighbourhoods having the same form as the sets $N_{\eta, d}(z, x)$.

We define a mapping $\pi: R^{*} \times X(s) \rightarrow X(s)$ in such a way that $\pi(t,\langle u, x\rangle) \stackrel{\text { df }}{=}\left\langle z_{x+t}, x+t\right\rangle$, where $z \in \mathscr{D}[s]$ is such that $z_{x}=u$. We observe, as in Section 3.2, that $\left(X(s), R_{*}: \pi\right)$ is a GSD-system. 
Now, we define

(6.26) $\quad M(s)$

$=\left\{\langle u, x\rangle \in X(s):\left|u_{(t)}(t, y)\right|<d-c t\right.$ for every $\left.t \in[0, \min (x, \delta))\right\}$.

Observe that

$$
(M(s))_{e}=\left\{\langle u, x\rangle \in X(s):\left|u_{x}(t, y)\right| \leqslant d-c \cdot \min (x, \delta)\right\} .
$$

It is obvious, that $M_{e}=M_{\text {se }}$ (we write shortly $M$ in the place of $M(s)$ ) and that $M_{s e}=\left\{\langle u, x\rangle:\left|u_{x}(x, y)\right|=d-c \cdot \min (x, \delta)\right.$ for some $y$ and $\left\langle u_{t}, t\right\rangle$ belongs to $M(s)$ for $\left.0 \leqslant t<x\right\}$. This is a corollary of $(\mathrm{A})$.

Moreover, the set $S^{0}(s)=\{0\} \times \mathscr{P}^{0} \subset M \cup M^{*}$ is such that, there are no retractions from $S^{0}(s)$ onto

$$
\left.S^{0}(s) \cap\left(M_{e} \cup M^{*}\right)=\{\dot{\langle} u, 0\rangle: u_{0} \epsilon \mathscr{P}^{0},\left|u_{0}(0, y)\right|=d \text { for some }|y| \leqslant \mu\right\} .
$$

This follows directly from Corollary 3 in Section 3.2.

But there is a retraction of $M_{e} \cup M^{*}$ onto $S^{0}(s) \cap\left(M_{e} \cup M^{*}\right)$. It is given by the formula

$$
M_{e} \cup M^{*} \ni\langle u, x\rangle \mapsto\left\langle w_{x}, 0\right\rangle \epsilon \mathbb{S}^{0}(s) \cap\left(M_{e} \cup M^{*}\right),
$$

where the function $w$ is such that $w(0, y)=w^{v, x}(y)$ with $v=u_{x}$.

In order to verify the correctness of the above definition we refer to Proposition 1.

Hence, all assumptions of Theorem $\mathrm{R}$ from Part $\mathrm{I}$ (Section 8 ) are satisfied (the fact that $M$ is open is trivial). Then, we can apply this theorem; the conclusion of Theorem $R$ means that the conclusion of our Theorem holds true.

6.4. A modification of the main Theorem 8. We will use the notation introduced in Section $6.1(\mathrm{i})-6.1(\nabla)$ and we will assume all conditions supposed in Section 6.1 (i) -6.1 (iv).

THEOREM 9. Under the assumptions of 6.1 (i)-6.1 (iv) (that is under the assumptions of Theorem 8, possibly without condition (A)) and under the following condition

$$
\left\{\begin{array}{l}
|f(x, y, z, 0)| \neq c \text { for every }\langle x, y, z\rangle \text {, such that }\langle x, y, z, 0\rangle \text { belongs } \\
\text { to the domain of the definition of function } f,
\end{array}\right.
$$

there exists a function $\varphi \in \mathscr{P}$, such that $z=z[\varphi]$ fulfils the following ine. quality:

$$
\begin{gathered}
|z(x, y)|<d-c x, \quad \text { for }\langle x, y\rangle \text { belonging to the set } \\
\{\langle x, y\rangle: 0 \leqslant x<\delta,|y|<s(x)\} .
\end{gathered}
$$


CoROLLARY. Under the assumptions of Theorem 9, there is a function $\varphi \epsilon \mathscr{P}$. such that $z=z[\varphi]$ fulfils

$$
|z(x, y)| \leqslant d-c x \quad \text { for }\langle x, y\rangle \in Q(s) .
$$

Proof of Theorem 9. Let $\eta \epsilon(0, \delta)$ be fixed. We define, for every $x \in[0, \delta]$, a function $\mu_{x}:[0, x] \rightarrow R_{+}$by the following formula:

$$
\mu_{x}(t)=(1+t \cdot \alpha \cdot(1-\delta / x)) \cdot s(t) \quad(=(1-a t(\delta-x) / x) \cdot s(t)),
$$

with $s$ defined by (6.12), where $\alpha \in R_{+}$is fixed in such a way that $\alpha \cdot(\delta-\eta)<1$.

Of course, if $x<\xi$, then $\mu_{x}(t)<\mu_{\xi}(t)$ for $0<t \leqslant x$. We put:

$$
Q^{t}= \begin{cases}\left\{\langle x, y\rangle \in \hat{Q}: 0 \leqslant x \leqslant t,|y| \leqslant \mu_{\eta}(x)\right\} & \text { for } t \epsilon[0, \eta], \\ \left\{\langle x, y\rangle \in \hat{Q}: 0 \leqslant x \leqslant t,|y| \leqslant \mu_{t}(x)\right\} & \text { for } t \in[\eta, \delta), \\ \{\langle x, y\rangle \epsilon \hat{Q}: 0 \leqslant x \leqslant t,|y| \leqslant s(\delta)\} & \text { for } t \geqslant \delta .\end{cases}
$$

If $z^{0}=z[\varphi]$ is a solution of $(6.6)-(6.7)$. given by some $\varphi \in \mathscr{P}$, then we extend this function $z^{0}$ to the function $z$ defined on the whole set $Q[s]$ (see (6.24)) by formula (6.25).

Let $z$ be an extension of a solution $z^{0}=z[\varphi]$ of (6.6)-(6.7) and let $t$ be a number belonging to $R_{*}$. By $z^{t}$ we denote the restriction of $z$ to the set $Q^{t}$ given by (6.32). Denoting, as in Section 6.3 , by $\mathscr{D}[s]$ the family of all extensions $z$ of solutions $z^{0}=z[\varphi]$ of $(6.6)-(6.7)$, we put: $\hat{Z}=\hat{Z}(s)$ $\stackrel{\text { df }}{=}\left\{z^{t}: z \in \mathscr{D}[s], t \in R_{*}\right\}$ and we consider a subset $Y$ of $\hat{Z} \times R_{*}$ defined by the formula:

$$
\begin{aligned}
& Y=Y(s) . \\
& \stackrel{\mathrm{d} f}{=}\left\{\langle u, x\rangle: x \in R_{*}, \text { there exists } z \in \mathscr{D}[s] \text { such that } z^{x}=u\right\} .
\end{aligned}
$$

For $\langle z, x\rangle \epsilon \bar{Y}$ and $\langle\varepsilon, y\rangle \epsilon R_{+} \times R_{+}$we put

$$
\begin{aligned}
\hat{N}_{e, \gamma}(z, x) \stackrel{d f}{=}\{\langle u, t\rangle \in & Y: t \epsilon(x-\varepsilon, x+\varepsilon) \cap R_{*}, \\
& \max \left\{|\varphi(\xi, y)-\psi(\xi, y)|:\langle\xi, y\rangle \epsilon Q^{\max (t, x)}\right\}<\gamma, \\
& \text { for } \left.\varphi, \psi \in \mathscr{D}[s], \text { such that } \varphi^{x}=z, \psi^{t}=u\right\} .
\end{aligned}
$$

We can prove a lemma analogous to Lemma 1 , and then we can consider $\left\{\hat{N}_{\varepsilon \gamma}(z, x):\langle z, x\rangle \in Y,\langle\varepsilon, \gamma\rangle \in R_{+} \times R_{+}\right\}$as the fundamental family of neighbourhoods in $Y$; this means that open sets in $Y$ we define as unions of sets belonging to this family. Hence, we have defined in the set $Y$ a topology.

Let us put $T=R_{*}$ and consider the GSD-system $(Y, T, \pi)$ with $\pi: T \times Y \rightarrow Y$ defined by the formula:

$$
\pi(t, \dot{\langle} z, x\rangle) \stackrel{d f}{=}\left\langle\varphi^{x+t}, x+t\right\rangle \quad \text { with } \varphi \in \mathscr{D}[s] \text {, such that } \varphi^{x}=\dot{z} .
$$


The definition of $\pi$ is correct, in virtue of Theorem W. Moreover, $\pi$ is continuous; the proof is trivial.

By $M$ we denote the set of all $\langle z, x\rangle \in Y$, such that $x \in[0, \delta)$ and $\left|z^{x}(t, y)\right|<d-c t$ for $0 \leqslant t \leqslant x, y \in\left[-\mu_{x}(t), \mu_{x}(t)\right]$. Obviously $M$ is open. Furthermore $M_{e}=\left\{\langle z, x\rangle \in Y: 0<x<\delta,|z(t, y)|<d-c t\right.$ for $y \in\left(-\mu_{x}(t)\right.$, $\left.\mu_{x}(t)\right), t \in[0, x)$ and $\left|z^{x}(t, y)\right|=d$-ct for some $t \in[0, x)$ and some $y$, such that $|y|=\mu_{x}(t)$ or $\left|z^{x}(x, y)\right|=d-c x$ for some $y$, such that $\left.|y| \leqslant \mu_{x}(x)\right\}$.

It is not difficult to show, that condition (B) implies the following implication:

(I) if $\langle z, x\rangle \in M_{e}, z=(z[\varphi])^{x}$ for some $\varphi \in \mathscr{D}$, then there exists a positive number $\varrho$, such that the function

$$
(z[\varphi])^{x+e}: Q^{x+e} \rightarrow R
$$

satisfies the following condition: for every $t \epsilon(x, x+\varrho]$, there exists $\langle u, r\rangle \in Q^{t}$, for which

$$
\left|(z[\varphi])^{x+e}(u, r)\right|>d-c \cdot r .
$$

Indeed, if $|z(x, y)|=d-c x$ for some $y \in\left(-\mu_{z}(x), \mu_{x}(x)\right)$, then $\frac{\partial}{\partial y} z(x, y)=0$ (if not, then $\langle z, x\rangle$ can not belong to $\bar{M}$ ) and then $\frac{\partial}{\partial x} z(x, y)=f(x, y, z(x, y), 0)>c$ if $z(x, y)=d-c x$ and $\frac{\partial}{\partial x} z(x, y)<c$ if $z(x, y)=-d+c x$ (the opposite inequalities are impossible, because $\langle z, x\rangle \in \bar{M})$. Similarly, if $z\left(x-\varepsilon, \mu_{x}(x-\varepsilon)\right)=d-c(x-\varepsilon)$ for some $\varepsilon>0$ and $\frac{\partial}{\partial y} z\left(x-\varepsilon, \mu_{x}(x-\varepsilon)\right)<0$, we have a contradiction with the condition: $\langle z, x\rangle \epsilon \bar{M}$. Then $\frac{\partial}{\partial y} z\left(x-\varepsilon, \mu_{x}(x-\varepsilon)\right)>0$, or $\frac{\partial}{\partial y} z\left(x-\varepsilon, \mu_{x}(x-\varepsilon)\right)=0$ and then $\frac{\partial}{\partial x} z\left(x-\varepsilon, \mu_{x}(x-\varepsilon)\right)>c$.

In each of the above cases, the corresponding implication for the first partial derivative of $z$ with respect to the first or the second variable gives implication (I). The proof of $(I)$ in the other possible cases is similar.

Implication (I) means that $M_{e}=M_{s e}$.

Furthermore, $M^{\prime}=\{\langle z, 0\rangle:|z(0, y)|=d$ for some $y,|y| \leqslant s(0)\}$.

Using the notation from Sections 6.2 and 6.3. we have, as in Section 6.3 , for the set $S^{0}(s)=\{0\} \times \mathscr{P}^{0}$, the following properties:

(a) there are no retractions from $S^{0}(s)$ onto $S^{0}(s) \cap\left(M_{e} \cup M^{*}\right)$ $=\left\{\langle u, 0\rangle: u^{0} \epsilon \mathscr{P P}^{0},\left|u^{0}(0, y\rangle\right|=d\right.$ for some $\left.y \leqslant \mu\right\}$,

(b) there is a retraction of $M_{e} \cup M^{*}$ onto $S^{0}(s) \cap\left(M_{e} \cup M^{*}\right)$. 
The second assertion (b) we prove in the same manner as in the proof of Theorem 8. The only (inessential) change is that in the place of $w^{u, x}$ given by (6.16) we need here $\hat{w}^{u, x}(y)=d(d-c x)^{-1} \cdot u\left(\mu_{x}(x) \cdot \mu^{-1} \cdot y\right)$ for $y \in[-\mu, \mu]$, and for $\langle u, x\rangle \in M_{e} \cup M^{*}$ we replace $w^{v, x}$ by the above $\hat{w}^{v, x}$, with $v=u^{x}$ in the formula defining the required retraction.

Thus, all assumptions of Theorem $R$ from Section 8 in Part $I$ are satisfied. Hence, there exists a function $\varphi \in \mathscr{P}$, such that $z=z[\varphi]$, fulfils the inequality $|z(x, y)|\left\langle d-c \cdot x\right.$ for $\langle x, y\rangle \in Q^{t}$; in particular for $\langle x, y\rangle \epsilon$ $\epsilon Q^{i} \cap[0, \eta] \times R$. In order to finish the proof of the theorem we apply the same reasoning as in the case of Theorem 3 (Section 3.3), tending with $\eta$ to $\delta$. We omit the technical detailes.

6.5. Supplementary remarks. Some applications of Theorem $R$, similar to those given in Sections 6.2-6.4, one can find in the author's paper [25]. There are given results on partial differential equations of the first order in unbounded regions. The method and the general idea is the same as in the present paper. The author hopes that some applications for partial differential equations of the second oredr will be given separately.

\section{References}

[1] F. Albrech t, Remarques sur un théorème de $T$. Ważewski relatif à l'allure assymptotique des équations différentielles, Bull. Acad. Polon. Sci. Cl. III 2 (1954), p. $315-318$.

[2] P. N. Bajaj, Start points in semi-dynamical systems, Funkcialaj Ekvacioj, 13 (1971), p. 171-177.

[3] J. W. Bebernes and J. D. Schuur, The Wazewski topological method for contingent equations, Ann. Mat. Pura Appl. 87 (1970), p. 271-280.

[4] - Investigations in the topological method of Wazewski, Rend. Accad. Lincei, Sci. fis., mat. e nat. 49 (1970), p. 39-42.

[5] N. P. Bhatia and O. Hajek, Local semi-dynamical systems, Lecture Notes in Mathematics, New York 1969.

[6] N. P. Bhatia and G.P. Szegö, Dynamical systems: stability theory and applications, Lecture Notes in Mathematics, Berlin-Heidelberg-New York 1967.

[7] A. Bielecki, Extension de la méthode de retract de T. Ważewski aux équations du paratingent, Ann. Univ. M. Curie-Skłodowska, Sec. A, 9 (1955), p. 37-58.

[8] - and C. Kluczny, Sur un théorème concernant les systèmes d'équations différentielles ordinaires, ibidem 14 (1960), p. 117-125.

[9] K. Borsuk, Über die Abbildungen der metrischen kompakten Räume auf die Kreislinie, Fund. Math. 20 (1933), p. 224.

[10] И. Ү. Бронштан̆ (I. U. Bronshtain), О динамических системах без единственности как полугруппах неоднозначных отображений топологичвсково nространства (On dynamical systems without uniqueness as semigroups of multivalued mappings of a topological space), Д. A. H. C.C.C.P. 114 (1962) (Dokl. Akad. Nauk. SSSR, vol. 114 (1962)), p. 954-957. 
[11] В. Д. Федоров (V.D. Fedorov), Об аналоге monологического принчипа T. Важсевского для интегро-дифференчиальньх уравнений (On an analog of the topological principle of $T$. Wazewski for integrodifferential equations), Иcследования по интегро-дифф. уравн. в Киргизии (Issledovanija po integro-diff. uravn. จ Kirgizii), № 1, Фрунве (Frunze), A. H. Кирг. CCP (A. N. Kirg. SSR), (1961), p. 111-132.

[12] J. K. Hale and E. F. Infante, Extended dynamical systems and stability theory, Proc. Nat. Acad. Sci. U.S.A. 58 (1967), p. 405-409.

[13] J. L. Kaplan, A. Lasota and J.A. Yorke, An application of the Wazewski retract method to boundary value problems, Univ. of Maryland, Inst. for Fluid Dynamics and Appl. Math., Technical Note BN-667 (August 1970).

[14] C. Kluczny, Sur certaines familles de courbes en relation avec la théorie des équa. tions différentielles ordinaires. I, Ann. Univ. M. Curie-Skłodowska, Sect. A, 15 (1960), 13-40.

[15] - Sur certaines familles de courbes en relation avec la théorie des équations différentielles ordinaires. II, ibidem 16 (1962), p. 5-18.

[16] - On the characteristic exponents of the solution of a system of ordinary differential equations, Ann. Polon. Math. 7 (1960), p. 215-239.

[17] - On the asymptotic behaviour of the solutions of a system of ordinary differential equations, ibidem 12 (1963), p. 257-263.

[18] S. Lojasiewicz, Sur l'allure asymptotique des intégrales du système d'équations différentielles au voisinage du point singulier, ibidem 1 (1954), p. 34-72.

[19] Z. Mikolajsika, Remarque sur la structure de l'ensemble engendré par les intégrales asymptotiques d'un système d'équations différentielles, Colloq. Math. 6 (1958), p. 219-221.

[20] C. Olech, On the asymptotic behaviour of the solutions of a system of ordinary non. linear differential equations, Bull. Acad. Polon. Sci. Cl. III, 4 (1956).

[21] N. Onuchic, Applications of the topological method of Wazewstei to certain problems of asymptotic behaviour in ordinary differential equations, Pacific J. Math. 11 (1961), p. 1511-1527.

[22] A. Pelczar, On some extensions of the retract theorem of $T$. Wazewski, Bull. Acad. Polon. Sci., Sér. Sci. Math., Astronom. Phys. 17 (1969), p. 693-698.

[23] - On some extensions of the retract theorem of T. Wasezwski. II, ibidem 18 (1970), p. 641-647.

[24] - On some extensions of the retract theorem of T. Ważewski. III, ibidem 19 (1971), p. $445-449$.

[25] - On some extensions of the retract theorem of T. Waziewski, IV, ibidem 20 (1972), p. 145-152.

[26] - Some functional differential equations, Diss. Math. (Rozprawy Mat.) 100 (1972).

[27] - A theorem on some extended dynamical systems, Congrés International des Mathématiciens; Nice 1970, Sect. $D_{12}$, p. 238 (Communications Indyviduelles).

[28] A. Plis, On a topological method for studying the behaviour of the integrals of ordinary differential equations, Bull. Acad. Polon. Sci., Cl. III, 2 (1954), p. 415-418.

[29] E. Roxin, On generalized dynamical systems defined by contingent equations, J. Diff. Equations 1 (1965), p. 188-205.

[30] R. Sikorski, Funkcje rzeczywiste I (Real functions I), Monografie Mat., T. 35, PWN, Warszawa 1958.

[3i] V. A. Staikos, On the asymptotic behavior of solutions of perturbed linear systems of ordinary differential equations [in Greek; English summary], Second (Postdoctoral) Thesis at the Faculty of Sciences of the University of Athens, Athens 1967. 
[32] J. Szarski, Characteristics and Cauchy problem for non-linear partial differential equations of first order, University of Kansas, Department of Mathematics, Studies in Eigenvalue Problems, Technical Report 21, Lawrence, Kansas 1959. [33] - Differential inequalities, PWN, Warszawa 1965.

[34] - et T. Ważewski, Sur l'existence des intégrales asymptotiques des équations différentielles issues d'un ensemble de dimension zéro, Colloq. Math. 6 (1958), p. 215-218.

[25] Z. S z my dt, Sur l'allure asymptotique des intégrales des équations différentielles ordinaires, Ann. Soc. Polon. Math. 24 (1951), p. 17-34.

[36] K. Tatarkiewicz, Propriétés asymptotiques des systèmes d'équations différentielles ordinaires presque linéaires, Ann. Univ. M. Curie Skłodowska 8 (1954), p. 25-69.

[37] T. Wa żewski, Sur un principe topologique de l'examen de l'allure asymptotique des intégrales des équations différentielles ordinaires, Ann. Soc. Polon. Math. 20 (1947), p. 279-313.

[38] - Sur les intégrales asymptotiques des équations différentielles ordinaires, Co. R. Soc. Sci. et Lett., Varsovie, Cl. III (1947), p. 38-42.

[39] - Utber die Bedingungen der Existenz der Integrale partieller Differentialgleichungen erster Ordnung, Math. Z. 43 (1938), p. 522-532.

[40] - Sur l'appréciation de domain d'existence des intégrales de l'équations aux dé. rivées partielles du premier ordre, Ann. Soc. Polon. Math. 14 (1935), p. 149-177.

[41] - Une méthode de l'examen du phénomène asymptotique relativement aux équations différentielles ordinaires, Rend. Accad. Lincei (8), 3 (1947), p. 210-215.

[42] - Sur une méthode topologique de l'examen de l'allure asymptotique des intégrales des équations différentielles, Proc. Internat. Congr. Mathematiciens, 1954, Groningen-Amsterdam (1956), p. 132-139.

Reģu par la Rédaction le 15. 6. 1972 


$$
\text { - }
$$

